\title{
TMT-based quantitative proteomic analysis reveals defense mechanism of wheat against the crown rot pathogen Fusarium pseudograminearum
}

Fangfang Qiao ${ }^{1+}$, Xiwen Yang ${ }^{1 \dagger}$, Fengdan Xu' ${ }^{1}$, Yuan Huang ${ }^{1}$, Jiemei Zhang ${ }^{1}$, Miao Song ${ }^{1}$, Sumei Zhou ${ }^{1}$, Meng Zhang ${ }^{2^{*}}$ and Dexian $\mathrm{He}^{1 *}$

\begin{abstract}
Background: Fusarium crown rot is major disease in wheat. However, the wheat defense mechanisms against this disease remain poorly understood.

Results: Using tandem mass tag (TMT) quantitative proteomics, we evaluated a disease-susceptible (UC1110) and a disease-tolerant (PI610750) wheat cultivar inoculated with Fusarium pseudograminearum WZ-8A. The morphological and physiological results showed that the average root diameter and malondialdehyde content in the roots of PI610750 decreased 3 days post-inoculation (dpi), while the average number of root tips increased. Root vigor was significantly increased in both cultivars, indicating that the morphological, physiological, and biochemical responses of the roots to disease differed between the two cultivars. TMT analysis showed that 366 differentially expressed proteins (DEPs) were identified by Gene Ontology and Kyoto Encyclopedia of Genes and Genomes enrichment in the two comparison groups, UC1110_3dpi/UC1110_0dpi (163) and PI610750_3dpi/PI610750_0dpi (203). It may be concluded that phenylpropanoid biosynthesis (8), secondary metabolite biosynthesis (12), linolenic acid metabolites (5), glutathione metabolism (8), plant hormone signal transduction (3), MAPK signaling pathway-plant (4), and photosynthesis (12) contributed to the defense mechanisms in wheat. Protein-protein interaction network analysis showed that the DEPs interacted in both sugar metabolism and photosynthesis pathways. Sixteen genes were validated by real-time quantitative polymerase chain reaction and were found to be consistent with the proteomics data.
\end{abstract}

Conclusion: The results provided insight into the molecular mechanisms of the interaction between wheat and $F$. pseudograminearum.

Keywords: Wheat (Triticum aestivum L.), Crown rot, Fusarium pseudograminearum, TMT, Differentially expressed proteins (DEPs), Defense mechanism

\footnotetext{
* Correspondence: zm2006@126.com; hed@henau.edu.cn

${ }^{\dagger}$ Fangfang Qiao and Xiwen Yang contributed equally to this work.

${ }^{2}$ College of Plant Protection, Henan Agricultural University, Zhengzhou

450002, Henan, China

'College of Agronomy, Henan Agricultural University/ National Engineering Research Center for Wheat/ Co-construction State Key Laboratory of Wheat and Maize Crop Science/ Collaborative Innovation Center of Henan Grain

Crops, 15 Longzihu College District, Zhengzhou 450046, China
}

(c) The Author(s). 2021 Open Access This article is licensed under a Creative Commons Attribution 4.0 International License, which permits use, sharing, adaptation, distribution and reproduction in any medium or format, as long as you give appropriate credit to the original author(s) and the source, provide a link to the Creative Commons licence, and indicate if changes were made. The images or other third party material in this article are included in the article's Creative Commons licence, unless indicated otherwise in a credit line to the material. If material is not included in the article's Creative Commons licence and your intended use is not permitted by statutory regulation or exceeds the permitted use, you will need to obtain permission directly from the copyright holder. To view a copy of this licence, visit http://creativecommons.org/licenses/by/4.0/ The Creative Commons Public Domain Dedication waiver (http://creativecommons.org/publicdomain/zero/1.0/) applies to the data made available in this article, unless otherwise stated in a credit line to the data. 


\section{Background}

Wheat (Triticum aestivum L.) is a major global food crop. Fusarium crown rot (FCR), caused by Fusarium pseudograminearum, is a major threat to wheat production. As one of the most devastating plant pathogens among soilborne diseases, F. pseudograminearum can absorb nutri-

ents from major winter cereals upon colonization [1]. The colonization of F. pseudograminearum initiates through epidermal penetration, most often through stomatal apertures, and progresses into the parenchymatous hypoderm. Hyphae spread from the culm base vertically through the tissues, initially through the hypoderm and pith cavity in culm tissues [2]. This pathogen mainly affects wheat, durum wheat (Triticum turgidum L. spp. durum (Dest.)), and barley (Hordeum vulgare L.) [3]. Although oats (Avena sativa L.) can be infected, they show few or no symptoms of disease [4]. In the Pacific Northwest and Australia, yield losses can reach up to $10-35 \%$ under natural inoculum levels [5-7]. According to reports, F. pseudograminearum also causes wheat crown rot in China, and in Henan, which is the largest wheat production province, the environmental conditions are especially suitable for F. pseudograminearum. This pathogen may present a serious threat to wheat production in the future [8]. Fully disease-resistant or immune cultivars have not been found in common wheat. Therefore, improving the genetic resistance of wheat to crown rot is an important objective.

Previous studies constructed a genetic map of F. pseudograminearum and completed a genomic sequence [9, 10]. Zhou et al. investigated the distribution and diversity of the pathogens associated with Fusarium crown rot in the Huanghuai wheat-growing region of China and found that F. pseudograminearum was the dominant species [11]. Several studies have confirmed that ER Lumenal Hsp70 protein FpLhs1, transcription factor FpAda1, and FpNPS9 are important for F. pseudograminearum infection [12-14]. FCR resistance responses in wheat are complex and controlled by multiple quantitative trait loci (QTLs) [15]. Thus, some studies have focused on the identification of gene loci in wheat and barley that are resistant to crown rot $[16,17]$. For instance, Yang et al. used a bi-parental population derived from the wheat cultivars UC1110 and PI610750 and detected three QTLs on chromosome 6A [18]. In addition, it has been reported that F. pseudograminearum produces a new class of active cytokinin that could activate plant cytokinin signaling during infection [19]. These molecules may extensively reprogram the host environment through crosstalk with defense hormone signaling pathways [20, 21].

With regard to plant defense responses triggered by $F$. pseudograminearum, host-pathogen interactions have been studied by transcriptome analyses in wheat using an Affymetrix gene chip [22]. It has been suggested that the differentially expressed genes are involved in antibacterial defense, oxidative stress, and signal transduction, as well as in primary and secondary metabolism [22]. Some defense-related genes were also found to be induced more rapidly in the FCR-resistant cultivar

Sunco than in the susceptible cultivar Kennedy [23]. In addition, many of the F. pseudograminearum-responsive genes are altered by toxin deoxynivalenol and plant defense-related hormones, which prevent $F$. pseudograminearum infection in wheat plants [22]. A combination of transcriptomics and metabolomics also has been used to study defense responses, and genes related to pathogen recognition and signal transduction, transcription factors, cell transport, and detoxification have been discovered [24].

Currently, little is known about the dynamics of the proteome and metabolome in infected host plants, and the resistance to $F$. pseudograminearum in wheat has not yet been explored from a proteomics perspective. In this study, we selected wheat cultivars with tolerance and susceptibility to F. pseudograminearum as the research materials. We analyzed protein expression abundance in the wheat following F. pseudograminearum infection using tandem mass tag (TMT) quantitative proteomics technology. Our objectives were to clarify how these proteins participate in resistance and to gather information on the inducible defense mechanisms in response to F. pseudograminearum infection. We also expect that this study will provide a new perspective for germplasm innovation regarding resistance to $F$. pseudograminearum in wheat, as well as contribute to the genetic improvement and breeding of new cultivars.

\section{Results}

Impact of $F$. pseudograminearum stress on wheat growth and development

The results showed that $F$. pseudograminearum stress affected wheat seedling growth and development, especially in the root system (Fig. 1a-h). At 3 days post-inoculation (dpi), light brown symptoms of disease initially appeared on the stem bases of the susceptible cultivar UC1110, which indicated that the incubation period was over (Fig. 1a, b). According to our observations, the average root diameter of the PI610750 seedlings was significantly decreased by $11.0 \%$ at $3 \mathrm{dpi}$ compared with that of the untreated seedlings at $3 \mathrm{dpi}$ (CK) (Fig. 1f), while the average number of root tips in the PI610750 seedlings was significantly increased by $30.0 \%$ (Fig. 1g). However, the total root length, total root surface area, total root volume, and forks of the UC1110 and PI610750 seedlings did not differ significantly (Fig. 1c-e, h). 
These physiological results showed that the root vigor of both the UC1110 and PI610750 wheat seedlings was significantly increased by 20.4 and $40.5 \%$, respectively, at 3 dpi compared with that in CK (Fig. 2a), while the malondialdehyde (MDA) content in the roots of the PI610750 seedlings was significantly decreased by $13.3 \%$ (Fig. 2h). The contents of soluble sugar and protein in the roots, the leaf chlorophyll content, and the activity of superoxide dismutase (SOD), peroxidase (POD), and catalase (CAT) in the roots of the UC1110 and PI610750 seedlings did not differ significantly (Fig. $2 b-f$, g). These results indicated that there were certain differences in the morphological, physiological, and biochemical responses of the disease-tolerant cultivar PI610750 and disease-susceptible cultivar UC1110 to F. pseudograminearum stress.

\section{Identification of DEPs in response to $F$. pseudograminearum infection}

We comprehensively examined and identified the defense-related proteins of the stem bases of two wheat cultivars, UC1110 and PI610750, under F. pseudograminearum stress using TMT quantitative proteomics technology. We selected the stem bases of wheat for proteomic analysis in this experiment because they represent the first obstacle to the invasion of the crown rot pathogen $F$. pseudograminearum.
To investigate the mechanisms of the differences in resistance of UC1110 and PI610750 at the protein level following $F$. pseudograminearum inoculation, we compared two groups, that is, UC1110_3dpi/UC1110_0dpi and PI610750_3dpi/PI610750_0dpi, using TMT quantitative proteomics. Compared with the UC1110_0dpi treatment, we identified 163 differentially expressed proteins (DEPs) in the UC1110_3dpi treatment, including 75 up-regulated and 88 down-regulated protein species, of which 100 were specifically expressed in this group (Figs. 3 and 4). In PI610750_3dpi/PI610750_0dpi, 203 protein species were differentially expressed, containing 133 up-regulated and 70 down-regulated proteins, of which 140 were specifically expressed in this group. A total of 63 proteins were common in UC1110_3dpi/UC1110_0dpi and PI610750_3dpi/ PI610750_0dpi, including 23 up-regulated and 40 downregulated proteins in UC1110_3dpi/UC1110_0dpi and 22 up-regulated and 41 down-regulated proteins in PI610750_3dpi/PI610750_0dpi (Fig. 4).

\section{Cluster analysis of DEPs based on GO enrichment}

To determine their potential functions, we annotated 366 DEPs by Gene Ontology (GO). The GO functional enrichment analysis showed that 186, 126, and 161 protein species were enriched in biological processes, cellular components, and molecular function, respectively (Additional file 1).

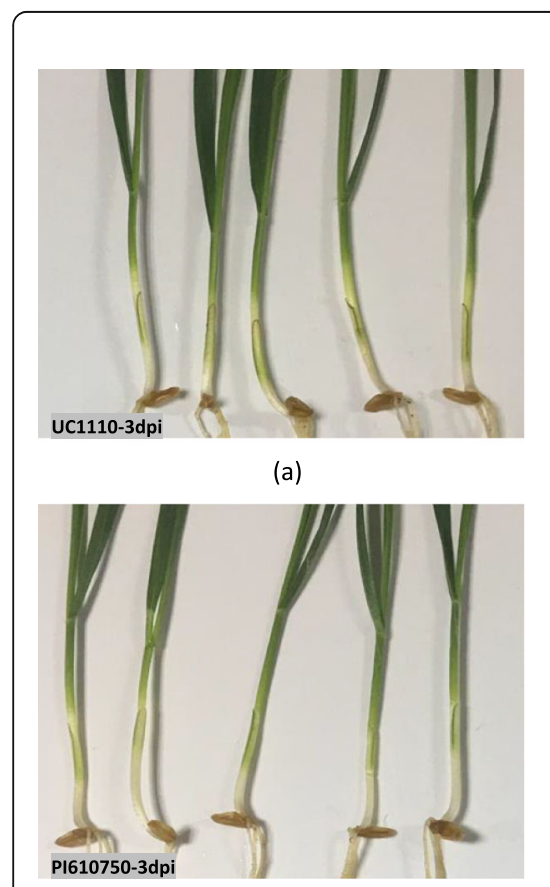

(b)

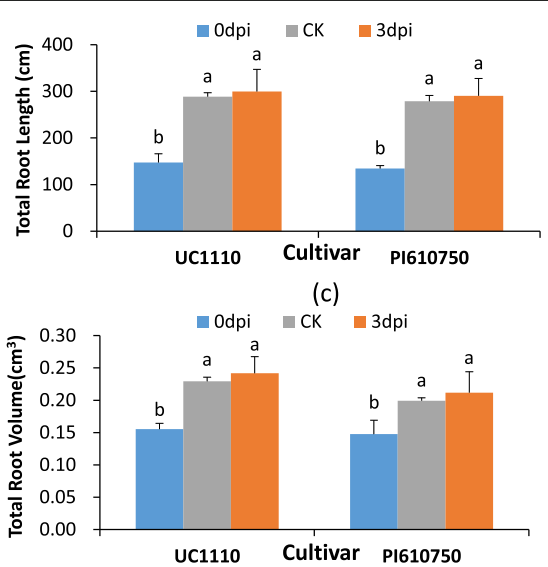

(e)

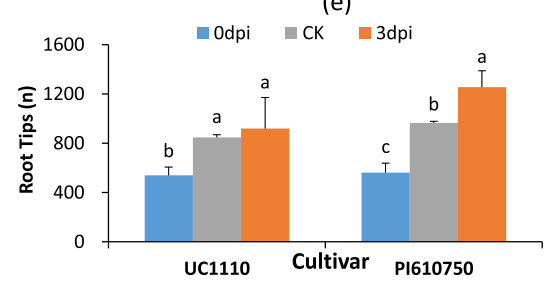

(g)

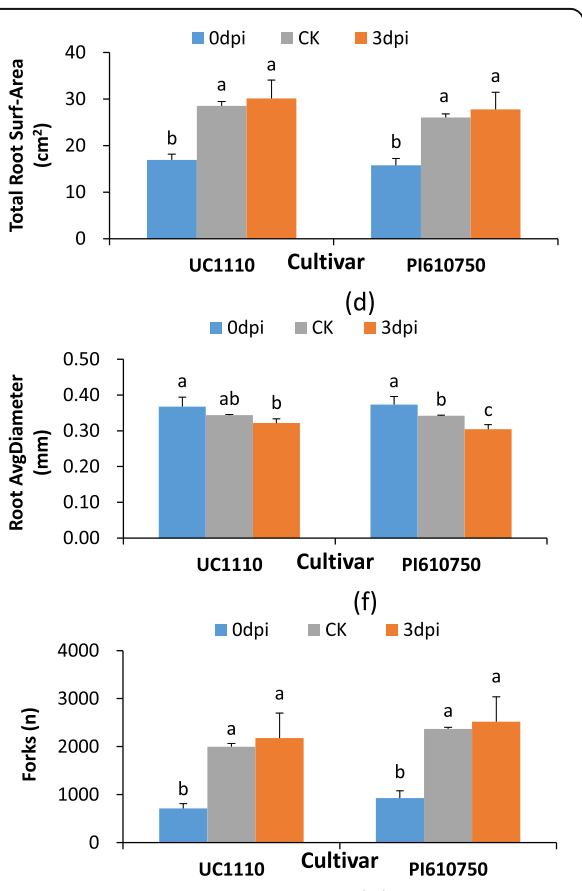

(h)

Fig. 1 Phenotypical and morphological parameters in the response to Fusarium pseudograminearum infection in wheat. Data are shown as mean \pm SD $(n=10)$ of three independent experiments. Different small letters ( $\mathbf{a}$ or $\mathbf{b})$ indicate a significant difference between the groups $(P<$ 0.05). CK: untreated 3 dpi sample 


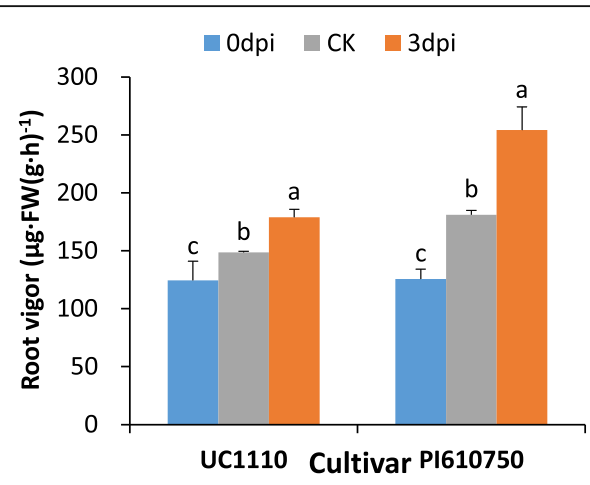

(a)

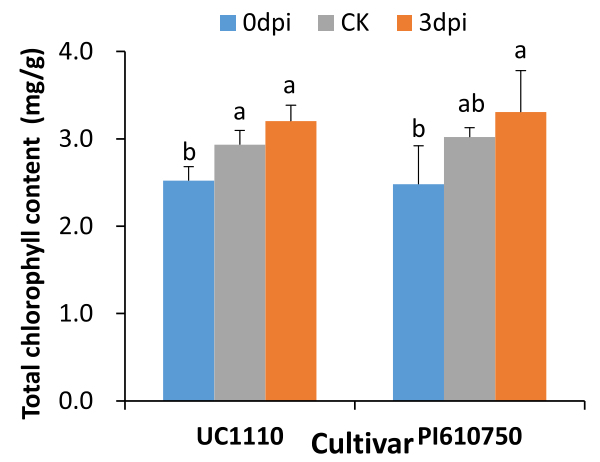

(c)

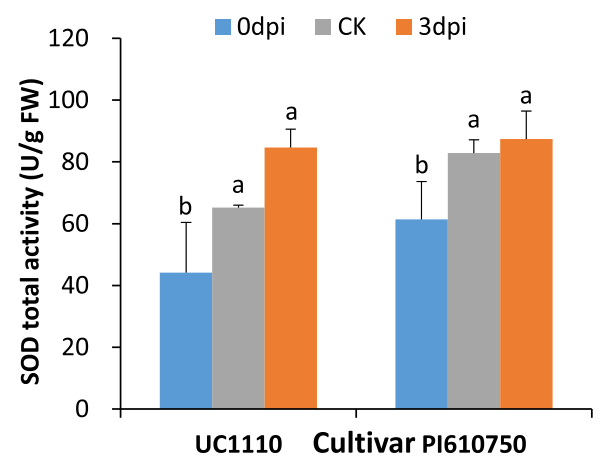

(e)

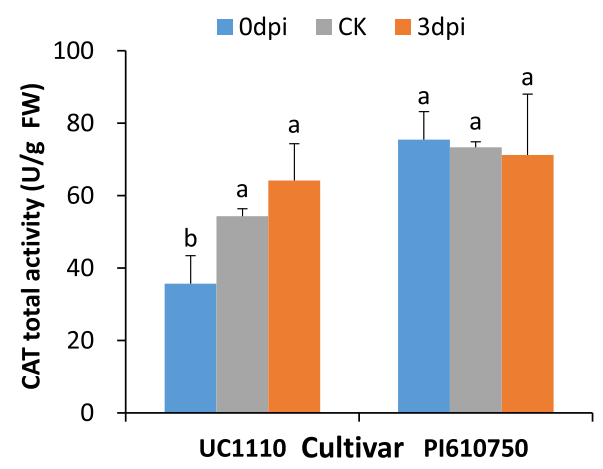

(g)

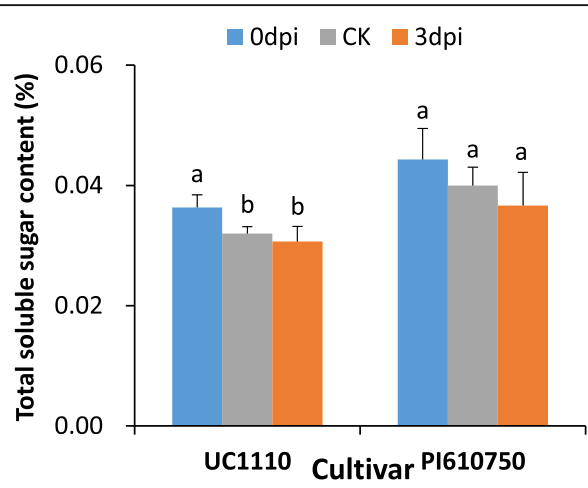

(b)

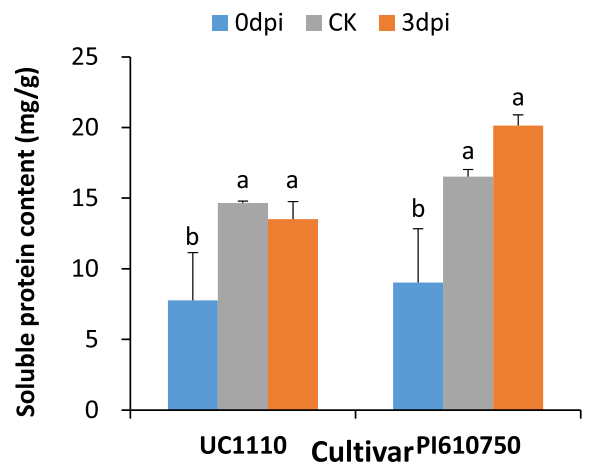

(d)

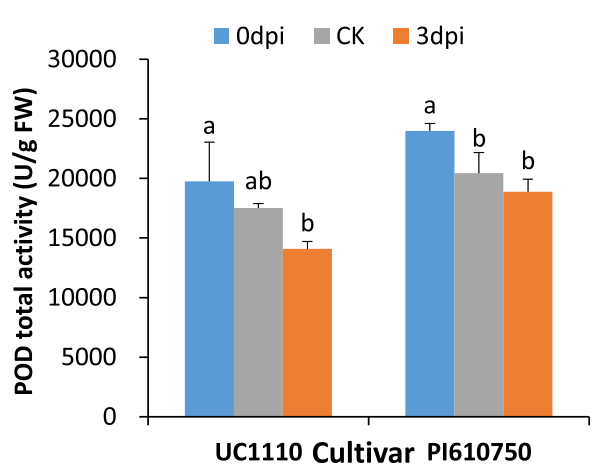

(f)

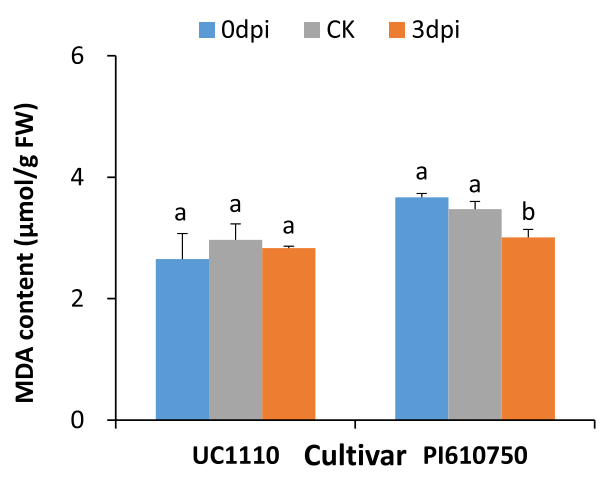

(h) 
(See figure on previous page.)

Fig. 2 Physiological and biochemical parameters in the response to F. pseudograminearum infection in wheat. Data are shown as mean \pm SD $(n=$ 4) of three independent experiments. Different lowercase letters $(\mathbf{a}$ or $\mathbf{b})$ indicate significant differences between the groups $(P<0.05)$. SOD: superoxide dismutase; POD: peroxidase; CAT: catalase; MDA: malondialdehyde. CK: untreated 3 dpi sample

\section{Enrichment of DEPs related to biological processes}

Under biological processes, the common DEPs $(\mathrm{C} 1)$ in the two comparison groups of UC1110_3dpi/UC1110_ Odpi and PI610750_3dpi/PI610750_0dpi were significantly enriched in the terms of organic acid catabolism, cell wall polysaccharide metabolism, and cell wall macromolecule metabolism (Fig. 5a). The specific DEPs (C2) of UC1110_3dpi/UC1110_0dpi were significantly enriched in the terms of negative regulation of hydrolase activity, dephosphorylation, regulation of proteolysis, negative regulation of protein metabolism, negative regulation of cellular metabolism, organic acid biosynthesis, carboxylic acid metabolism, oxoacid metabolism, and negative regulation of macromolecule metabolism (Fig. 5a). The specific DEPs (C3) of PI610750_3dpi/ PI610750_0dpi were significantly enriched in the terms of cellular protein metabolism, photosynthesis (dark reaction), carbohydrate biosynthesis, cellular macromolecule biosynthesis, the photosynthetic electron transport chain, polysaccharide biosynthesis, lipid transport, cellular carbohydrate biosynthesis, hexose metabolism, cellular polysaccharide metabolism, photosynthesis (light harvesting), amide biosynthesis, peptide biosynthesis, and peptide metabolism (Fig. 5a). This analysis showed that the DEPs related to organic acid catabolism and cell wall metabolism responded to $F$. pseudograminearum stress in the seedling stem bases of both the susceptible and tolerant cultivars. The disease-susceptible cultivar UC1110 also responded to stress through the DEPs related to dephosphorylation and carboxylic acid metabolism, while the disease-tolerant cultivar PI610750 mainly responded to stress through the DEPs related to photosynthesis and sugar metabolism.

\section{Enrichment of DEPs related to cellular components}

In the cellular components category, the common DEPs (C1) in the two comparison groups of UC1110_3dpi/ UC1110_0dpi and PI610750_3dpi/PI610750_0dpi were significantly enriched in the cell walls, external encapsulating structures, the cell periphery, and extracellular regions (Fig. 5b). The specific DEPs (C2) of UC1110_3dpi/ UC1110_0dpi were significantly enriched in the apoplasts (Fig. 5b). The specific DEPs (C3) of PI610750_ 3dpi/PI610750_0dpi were significantly enriched in the plastid thylakoids, chloroplast thylakoids, photosystem, photosynthetic membranes, cytoplasmic parts, thylakoid membranes, ribosomes, ribonucleoprotein complex, cytoplasm, membrane protein complex, and organelles (Fig. 5b). This analysis showed that the DEPs related to the cell wall first responded to $F$. pseudograminearum
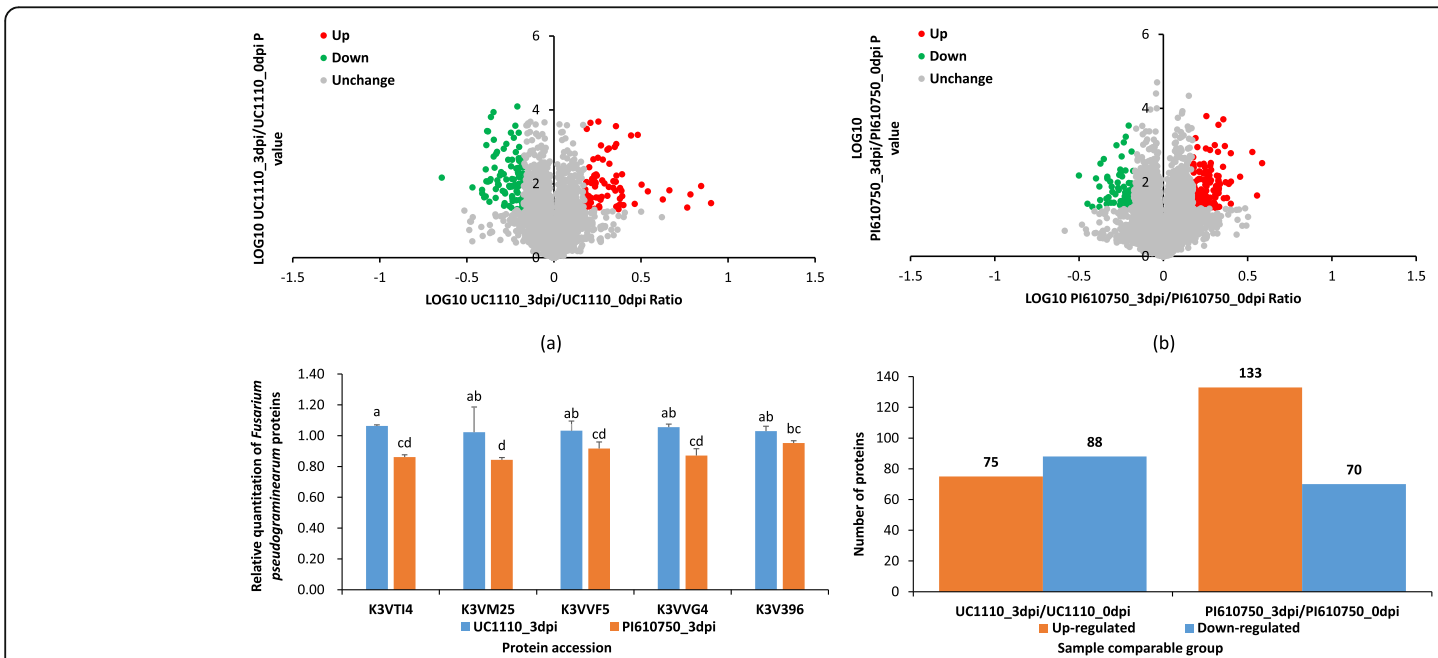

(c)

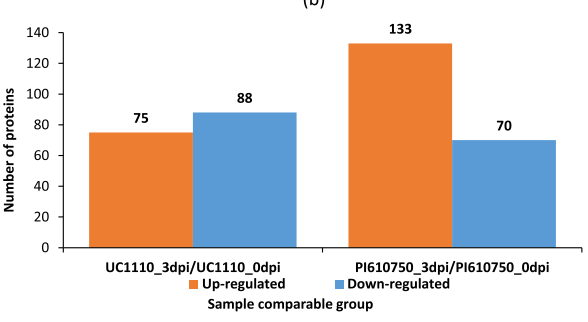

(d)

Fig. 3 Differentially expressed proteins (DEPs) analysis between UC1110_3dpi/UC1110_Odpi and PI610750_3dpi/PI610750_0dpi. Volcano plot of all DEPs in UC1110_3dpi/UC1110_Odpi (a) and PI610750_3dpi/PI610750_Odpi (b); relative quantitation analysis of five marker proteins of $F$. pseudograminearum between UC1110_3dpi and PI610750_3dpi (c). Data are shown as mean \pm SD $(n=3)$ of three independent experiments. Different lowercase letters $(\mathbf{a}$ or $\mathbf{b})$ indicate significant differences between the groups $(P<0.05)$. K3VTI4: Members of the aldo keto reductase family; K3VM25: NF-X1 finger and helicase; K3WF5: BHLH family transcription factor; K3WG4: BHLH family transcription factor; K3V396: nucleoporin; quantitative analysis of the proteome between UC1110_3dpi/UC1110_0dpi and PI610750_3dpi/PI610750_0dpi (d). In blue (downregulated): DEPs with $t$-test $P<0.05$ and fold-change $<0.667$; in orange (up-regulated): DEPs with $t$-test $P<0.05$ and fold-change $>1.5$ 


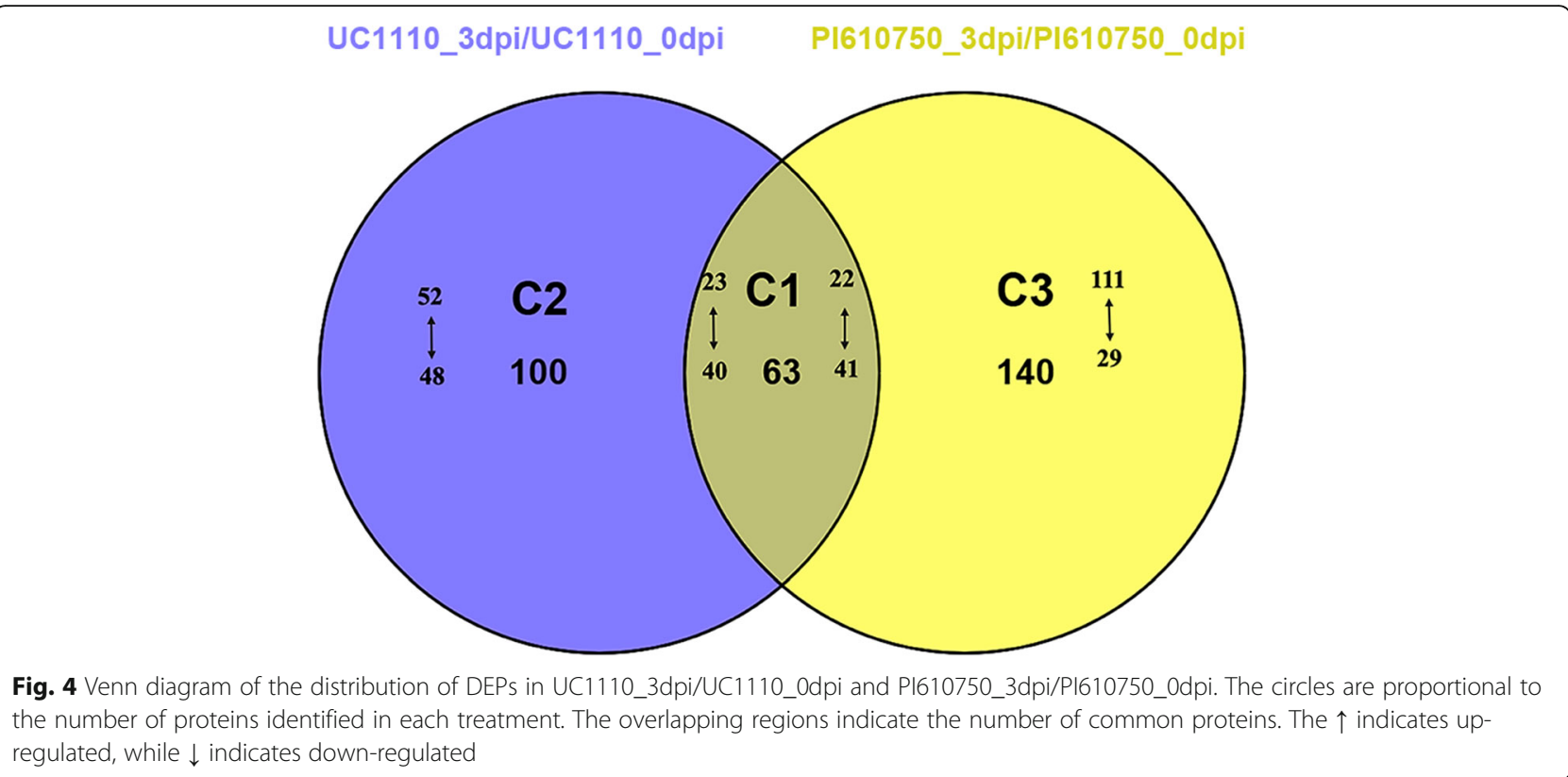

stress in the seedling stem bases of both the susceptible and tolerant cultivars. The disease-susceptible cultivar UC1110 also responded to stress through the DEPs related to apoplasts, while the disease-tolerant cultivar PI610750 mainly responded through the DEPs related to chloroplasts.

\section{Enrichment of DEPs related to molecular function}

In terms of molecular function, the common DEPs (C1) in the two comparison groups of UC1110_3dpi/ UC1110_0dpi and PI610750_3dpi/PI610750_0dpi were significantly enriched in the terms of glucosidase activity, hydrolase activity, phosphatidylcholine1-acylhydrolase activity, cysteine-type peptidase activity, phospholipase activity, lipase activity, and carboxylic ester hydrolase activity (Fig. 5c). The specific DEPs (C2) of UC1110_3dpi/ UC1110_0dpi were significantly enriched in the terms of the peptidase regulator activity, endopeptidase regulator activity, phosphatase activity, oxidoreductase activity, nitronate monooxygenase activity, flavin mononucleotide binding, hydrolase activity, enzyme inhibitor activity, transferase activity, dioxygenase activity, and acid phosphatase activity (Fig. 5c). The specific DEPs (C3) of PI610750_3dpi/PI610750_0dpi were significantly enriched in the terms of the electron transporter activity, electron carrier activity, ribulose-bisphosphate carboxylase activity,

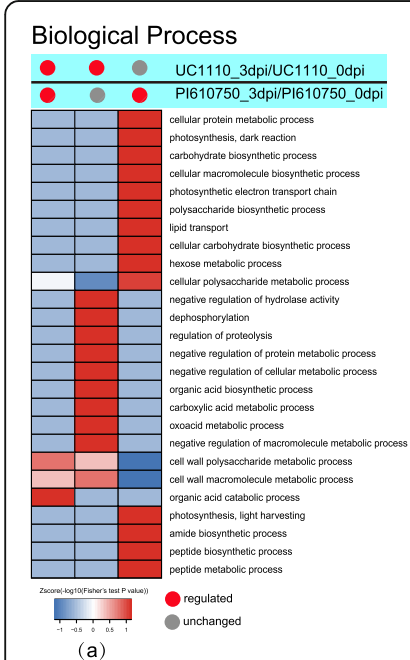

(a)

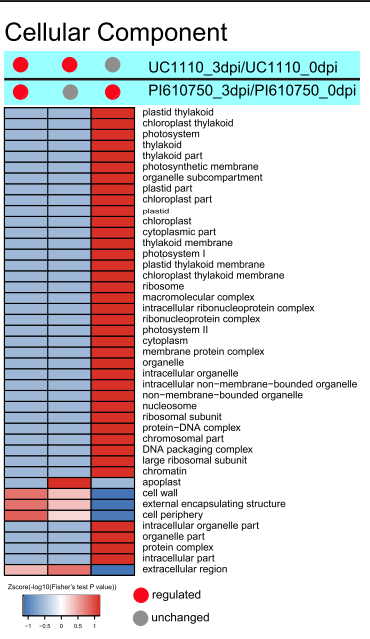

(b)

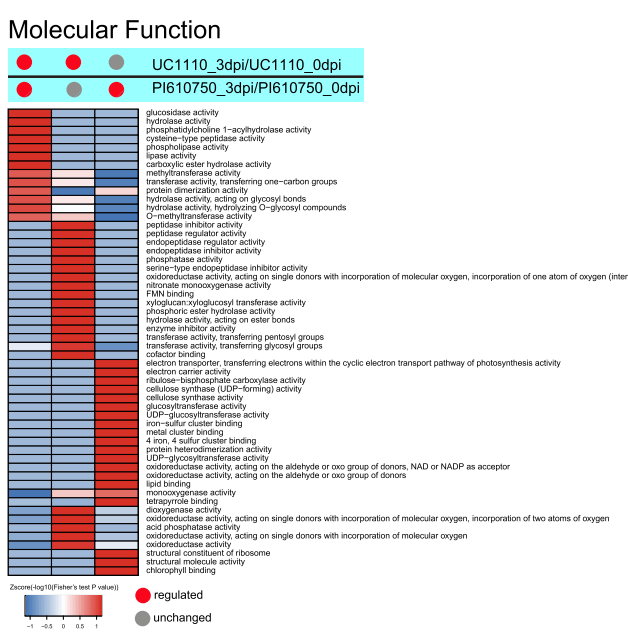

(c)

Fig. 5 GO-functional enrichment cluster analysis of DEPs: (a) biological process enrichment analysis; (b) cellular component enrichment analysis; (c) molecular functional enrichment analysis 
cellulose synthase activity, glucosyltransferase activity, metal cluster binding, protein heterodimerization activity, oxidoreductase activity, lipid binding, tetrapyrrole binding, structural constituents of ribosomes, structural molecule activity, and chlorophyll binding (Fig. 5c). This analysis showed that the disease-tolerant cultivar PI610750 mainly responded to stress through the DEPs related to electron transporter activity, electron carrier activity, cellulose synthase activity, and oxidoreductase activity in the plantpathogen interaction process.

\section{Kyoto encyclopedia of genes and genomes pathway} enrichment analysis of DEPs

Further analysis using Kyoto Encyclopedia of Genes and Genomes (KEGG) pathway enrichment showed that all DEPs of the two comparison groups were significantly enriched in the terms of ribosomes (20\%), phenylpropanoid biosynthesis $(14 \%)$, photosynthesis (11\%), glutathione metabolism (11\%), carbon fixation in photosynthetic organisms (7\%), alpha-linolenic acid metabolism (7\%), glyoxylate and dicarboxylate metabolism (7\%), linoleic acid metabolism (6\%), cyanoamino acid metabolism (6\%), photosynthesis-antenna proteins (6\%), and flavone and flavonol biosynthesis (5\%) (Additional file 2). The 63 common DEPs $(\mathrm{C} 1)$ in the two comparison groups of UC1110_3dpi/UC1110_0dpi and PI610750_3dpi/PI610750_ Odpi were significantly enriched in the terms of biosynthesis of secondary metabolites (osa01110), phenylpropanoid biosynthesis (osa00940), protein processing in endoplasmic reticulum (osa04141), starch and sucrose metabolism (osa00500), and cyanoamino acid metabolism (osa00460) (Fig. 6, Additional file 3).

\section{KEGG pathway}

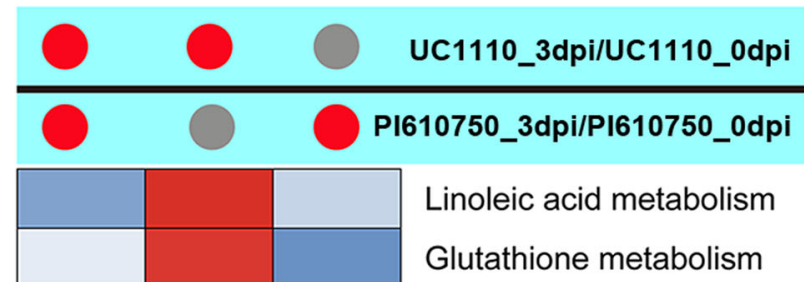

Ubiquinone and other terpenoid-quinone biosynthesis

Plant hormone signal transduction

MAPK signaling pathway - plant

alpha-Linolenic acid metabolism

Carbon fixation in photosynthetic organisms

Glyoxylate and dicarboxylate metabolism

Flavone and flavonol biosynthesis

Protein processing in endoplasmic reticulum

Biosynthesis of secondary metabolites

Starch and sucrose metabolism

Cyanoamino acid metabolism

Phenylpropanoid biosynthesis

Ribosome

Photosynthesis

Photosynthesis - antenna proteins

Zscore(-log10(Fisher's test $P$ value))

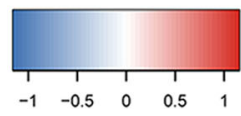

regulated

unchanged

Fig. 6 KEGG pathway enrichment cluster analysis of the DEPs of the two comparison groups 
The specific DEPs (C2) of UC1110_3dpi/UC1110_0dpi were significantly enriched in the terms of linoleic acid metabolism (osa00591), glutathione metabolism (osa00480), alpha-linolenic acid metabolism (osa00592), MAPK signaling pathway-plant (osa04016), flavone and flavonol biosynthesis (osa00944), and ubiquinone and other terpenoid-quinone biosynthesis (osa00130) (Fig. 6, Additional file 4). The specific DEPs (C3) of PI610750_3dpi/PI610750_0dpi were significantly enriched in the terms of ribosomes (osa03010), photosynthesis (osa00195), photosynthesis-antenna proteins (osa00196), flavone and flavonol biosynthesis (osa00944), carbon fixation in photosynthetic organisms (osa00710), and glyoxylate and dicarboxylate metabolism (osa00630) (Fig. 6, Additional file 5). This analysis showed that the DEPs related to secondary metabolites, protein processing, and energy metabolism pathways responded to stress in the seedling stem bases of both the susceptible and tolerant cultivars. The susceptible cultivar UC1110 responded to stress mainly through the DEPs related to linoleic acid metabolism and glutathione metabolism, and the disease-tolerant cultivar PI610750 mainly responded through the DEPs related to photosynthesis and glyoxylic acid and dicarboxylate metabolism.
Interaction network analysis of $F$. pseudograminearumresponsive proteins in wheat

The present study used the online STRING database and Cytoscape software to construct a protein-protein interaction network for all DEPs of the two comparison groups in response to $F$. pseudograminearum. This network showed that 76 of the possible DEPs interacted. With the MCODE plugin toolkit, three enriched interaction clusters were associated with ribosomes, photosynthesis, and sugar metabolism (Fig. 7). Sixteen interaction proteins belonged to the ribosome network. These proteins included 15 up-regulated proteins and one down-regulated protein in the comparison group PI610750_3dpi/PI610750_0dpi. Seven interaction proteins belonged to the carbohydrate metabolic process network, including four down-regulated proteins in the two comparison groups of UC1110_3dpi/UC1110_0dpi and PI610750_3dpi/PI610750_0dpi, and one down-regulated and two up-regulated proteins in the comparison group of PI610750_3dpi/PI610750_0dpi. Three interaction proteins belonged to the photosynthesis network, including three upregulated proteins in PI610750_3dpi/PI610750_0dpi. Further information about the proteins is shown in Additional file 6.

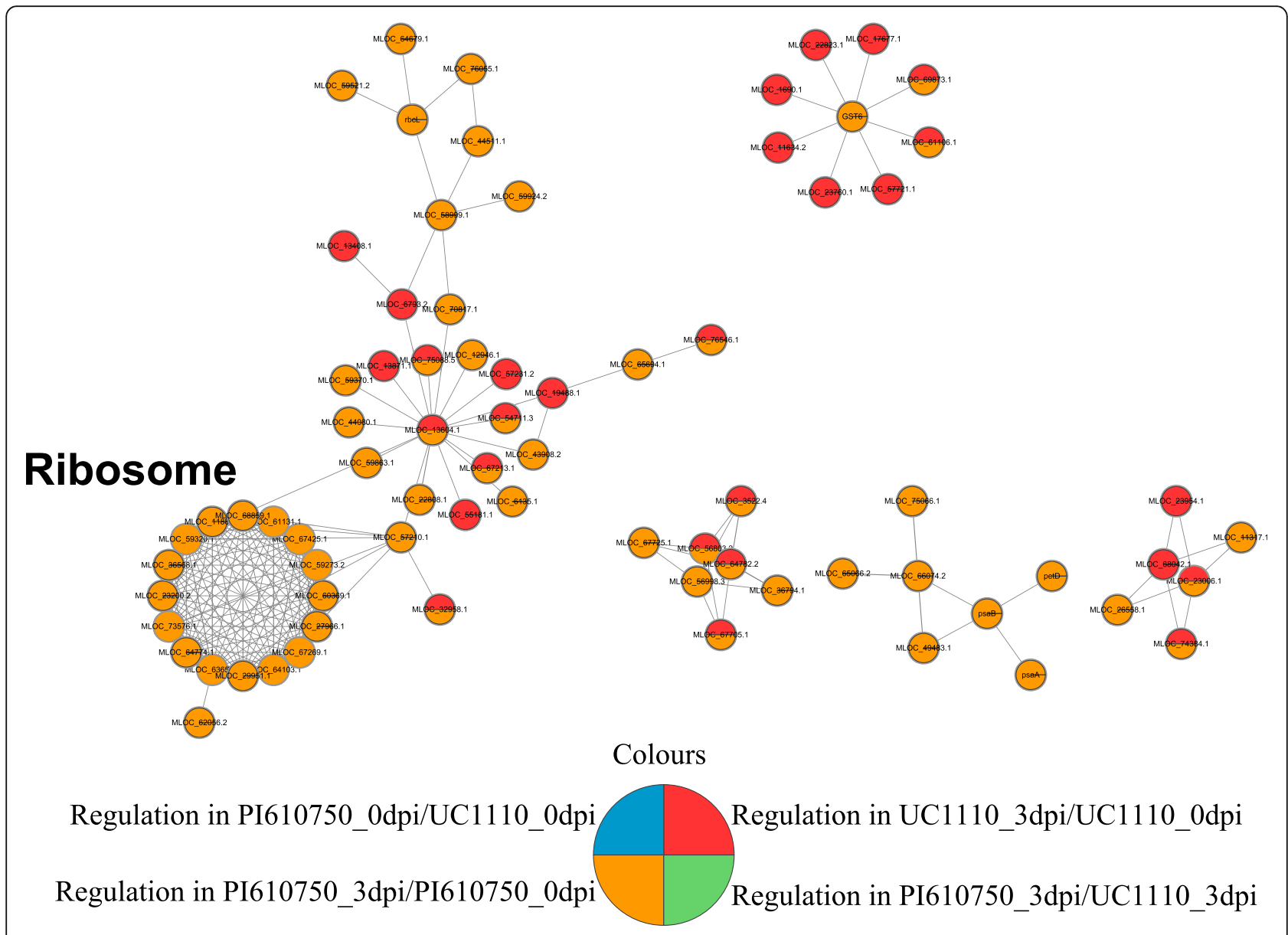

Fig. 7 Protein-protein interaction network analysis of the DEPs of the two comparison groups 
Correlation between mRNA and protein abundance

To further validate the reliability of the proteomics data, we selected 16 genes for quantitative real time-polymerase chain reaction (qRT-PCR) analysis. Three common genes in the two comparison groups showed similar tendencies as those for protein expression, including NMT1, GLU1B, and XIPI. In the comparison group of UC1110_3dpi/UC1110_0dpi, NMT1 and GLU1B were up-regulated and down-regulated, respectively. In the comparison group of PI610750_3dpi/PI610750_0dpi, however, both XIPI and GLU1B were down-regulated (Table 1). In the comparison group of UC1110_3dpi/ UC1110_0dpi, five specific DEPs were up-regulated at both the transcription and translation levels. Similarly, in the comparison group of PI610750_3dpi/PI610750_0dpi, eight specific DEPs were up-regulated at both the transcription level and translation level. The primer sequences for the 16 genes are listed in Additional file 7.

\section{Discussion}

The roots of the two wheat cultivars with different levels of disease tolerance showed obvious differences in their morphological, physiological, and biochemical responses to the crown rot pathogen F. pseudograminearum. Average root diameter and MDA content in the roots of the PI610750 seedlings decreased, while the average number of root tips increased. The increase in root vigor was higher at 3 dpi in PI610750 than in UC1110. This indicated that the defense mechanism of the wheat seedlings to the crown rot pathogen $F$. pseudograminearum was complex. Although many factors related to crown rot resistance have been identified, the molecular mechanisms of crown rot resistance are still poorly understood. Therefore, understanding the defense mechanism of wheat plants against crown rot is crucial to the sustainable improvement of wheat yield and quality. The DEPs of the two cultivars in this study were associated with metabolic pathways, plant-pathogen interaction, and photosynthesis.

\section{Metabolic pathways in response to $F$. pseudograminearum infection}

The metabolic pathways of the response of wheat to $F$. pseudograminearum were essential, accounting for $37 \%$ of DEPs in all KEGG pathways. Previous studies have shown that proline metabolism is implicated in the plant response to abiotic stress, and proline dehydrogenase (ProDH) is the first enzyme to catalyze the degradation of proline [25]. According to reports, 4-hydroxy-7-methoxy3-oxo - 3,4-dihydro-2H-1,4-benzoxazine-2-yl glucoside $\beta$ D-glucosidase is a typical member of multiple metabolic pathways, as 4-hydroxy-7-methoxy-3-oxo-3,4-dihydro$2 \mathrm{H}-1,4$-benzoxazin-2-yl glucoside $\beta$-D-glucosidase is involved in the metabolism of high-energy compounds and

Table 1 The mRNA and protein abundance changes of 16 selected genes in the study

\begin{tabular}{|c|c|c|c|c|c|c|c|c|}
\hline \multirow[t]{2}{*}{ Gene names } & \multicolumn{4}{|c|}{ UC1110-3dpi vs UC1110-0dpi } & \multicolumn{4}{|c|}{ PI610750-3dpi vs PI610750-0dpi } \\
\hline & $\begin{array}{l}\text { Gene fold } \\
\text { changes }\end{array}$ & $\begin{array}{l}\text { Regulated } \\
\text { type }\end{array}$ & $\begin{array}{l}\text { Protein fold } \\
\text { changes }\end{array}$ & $\begin{array}{l}\text { Regulated } \\
\text { type }\end{array}$ & $\begin{array}{l}\text { Gene fold } \\
\text { changes }\end{array}$ & $\begin{array}{l}\text { Regulated } \\
\text { type }\end{array}$ & $\begin{array}{l}\text { Protein fold } \\
\text { changes }\end{array}$ & $\begin{array}{l}\text { Regulated } \\
\text { type }\end{array}$ \\
\hline GLU1B & 0.25 & Down & 0.46 & Down & 0.15 & Down & 0.48 & Down \\
\hline NMT1 & 2.70 & Up & 1.68 & Up & 0.74 & Down & 1.78 & Up \\
\hline$X|P|$ & 1.46 & Up & 0.58 & Down & 0.34 & Down & 0.64 & Down \\
\hline cla30 & 2.41 & Up & 2.25 & Up & - & - & - & - \\
\hline gstu2 & 15.48 & Up & 2.27 & Up & - & - & - & - \\
\hline $\operatorname{Pr}-1-2$ & 4.18 & Up & 7.00 & Up & - & - & - & - \\
\hline Pr-1-1 & 8.43 & Up & 6.08 & Up & - & - & - & - \\
\hline PR-1.2 & 29.84 & Up & 2.35 & Up & - & - & - & - \\
\hline LIM & - & - & - & - & 2.21 & Up & 1.69 & Up \\
\hline $\mathrm{psaC}$ & - & - & - & - & 1.15 & Up & 2.13 & Up \\
\hline petD & - & - & - & - & 1.19 & Up & 1.66 & Up \\
\hline rps11 & - & - & - & - & 1.94 & Up & 1.56 & Up \\
\hline CENH3 & - & - & - & - & 2.44 & Up & 1.73 & Up \\
\hline Itp9.4b & - & - & - & - & 1.81 & Up & 1.51 & Up \\
\hline $\begin{array}{l}\text { TRAES_ } \\
\text { 3BF087500010CFD_C1 }\end{array}$ & - & - & - & - & 1.10 & Up & 2.51 & Up \\
\hline $\begin{array}{l}\text { TRAES_- } \\
\text { 3BF088300010CFD_C1 }\end{array}$ & - & - & - & - & 1.21 & Up & 2.51 & Up \\
\hline
\end{tabular}


plant growth [26]. Some studies have shown that $\beta$ glucosidase is involved in catalyzing the hydrolysis of glycosides to release glucose into the glycolysis process [27]. In plants, glutathione S-transferases (GSTs) have been shown to play a major role in cell detoxification and stress tolerance [28-30]. Previously, it was reported that lipoxygenase, allene oxide cyclase, and allene oxide synthase (AOS) are three important enzymes in jasmonic acid (JA) biosynthesis, and the activation of AOS enhances the drought tolerance of chickpea [31-33]. Studies have also shown that AOS transcripts and JA concentration in cells are critical for responses to pathogen and/or virus infections in plants $[34,35]$.

Some studies have indicated that NADH-dependent glutamate synthetase (NADH-GOGAT) is located in non-green tissues and is highly expressed in the roots, participates in the ammonium assimilation pathway, and promotes the absorption of nitrogen by plants [36]. Previous research has shown that the two enzymes phenylalanine ammonia lyase and cinnamoyl-CoA reductase are mainly involved in lignin biosynthesis. The biosynthesis of lignin is a major branch in the phenylpropane biosynthesis pathway, and the biosynthesis of phenylpropane is involved in the resistance of plants to diseases [37-39]. In this study, we found five common DEPs in the two comparison groups of UC1110_3dpi/UC1110 0dpi and PI610750_3dpi/PI610750_0dpi to be significantly down-regulated, including ProDH, 4-hydroxy-7methoxy-3-oxo-3,4-dihydro-2H-1,4-benzoxazin-2-yl glucoside beta-D-glucosidase, beta-glucosidase 26, AOS, and GSTU1. ProDH, AOS, and GSTU1 were enriched in the KEGG pathways of arginine and proline metabolism (osa00330), alpha-linolenic acid metabolism (osa00592), and glutathione metabolism (osa00480), respectively.

In addition, 4-hydroxy-7-methoxy-3-oxo-3,4-dihydro2H-1,4-benzoxazin-2-yl glucoside beta-D-glucosidase, and beta-glucosidase 26 were enriched in the three pathways of starch and sucrose metabolism (osa00500), cyanoamino acid metabolism (osa00460), and phenylpropanoid biosynthesis (osa00940) in the two comparison groups UC1110_3dpi/UC1110_0dpi and PI610750_3dpi/PI610750_0dpi. GSTU6 and NADHGOGAT were up-regulated and were enriched in the pathways of glutathione metabolism (osa00480) and nitrogen metabolism (osa00910), respectively. CinnamoylCoA reductase 1, peroxidase, and phenylalanine ammonia-lyase were also up-regulated and were enriched in the pathway of phenylpropanoid biosynthesis (osa00940). This indicated the following: (1) the ProDH and 4-hydroxy-7-methoxy-3-oxo-3,4-dihydro-2H-1,4benzoxazine-2-yl glucoside $\beta \mathrm{D}$-glucoside enzymes play an important role in the defense mechanism of wheat against F. pseudograminearum; (2) the study of GSTs in these two wheat cultivars may reveal the differences in the role of GSTU1 and GSTU6 in the defense mechanism of wheat against F. pseudograminearum; (3) the down-regulation of AOS in the JA pathway makes UC1110 more susceptible to pathogen infection; (4) cinnamoyl-CoA reductase 1, peroxidase, and phenylalanine ammonia lyase were up-regulated in the biosynthesis of phenylpropane, which is highly related to plant defense ability; and (5) the metabolic pathways of plants in response to pathogenic stress are complex and changeable (Fig. 8).

\section{Plant-pathogen interactions in wheat}

Plants have various defense mechanisms. These include the production of antimicrobial peptides, particularly pathogenesis-related proteins (PR proteins). PR proteins were first noted in plants as part of the hypersensitive response, but have since been assigned an array of biological roles [40]. PR proteins are a type of stress-responsive protein whose expression can be induced by pathogen invasion [41]. A number of studies have shown that PR proteins participate in plant defense mechanisms, as many of them are endowed with antimicrobial activity against plant pathogens, with different antifungal, antibacterial, and antiviral effects $[42,43]$. Regarding the specific DEPs in the comparison group of UC1110_3dpi/UC1110_0dpi, multiple PR proteins related to plant-pathogen interactions were identified, including PR protein-1.2, PR protein $1-1$, and PR protein $1-2$. The expression levels of these proteins were up-regulated in response to $F$. pseudograminearum infection. These proteins were up-regulated in PI610750_3dpi/PI610750_0dpi, but the difference was not significant.

PR protein 1 is an antimicrobial protein in host defense that is targeted by plant pathogens during infection [44-46]. The production of PR proteins in response to pathogen invasion is related to the plant disease resistance specialized in systemic acquired resistance (SAR) [47]. PR-1-5 is a potential target of ToxA, and the site-specific interaction between PR-1-5 and ToxA may mediate ToxA-induced necrosis of susceptible wheat [48]. In this study, pathogenesis-related (PR-1.2, Pr-1-1, $\mathrm{Pr}-1-2)$ proteins were up-regulated in the comparison group of UC1110_3dpi/UC1110_0dpi and were involved in plant hormone signal transduction (osa04075) and the MAPK signal plant pathway (osa04016), suggesting that the PR1 family plays an important role in the crown rot defense mechanism of wheat.

\section{Defensive photosynthetic activities of wheat stem bases under $F$. pseudograminearum infection}

In plants, chloroplast photosynthesis is an important biochemical reaction that converts light energy into chemical energy to maintain plant life [49]. Research on plant defense and photosynthesis has indicated that the rate of 


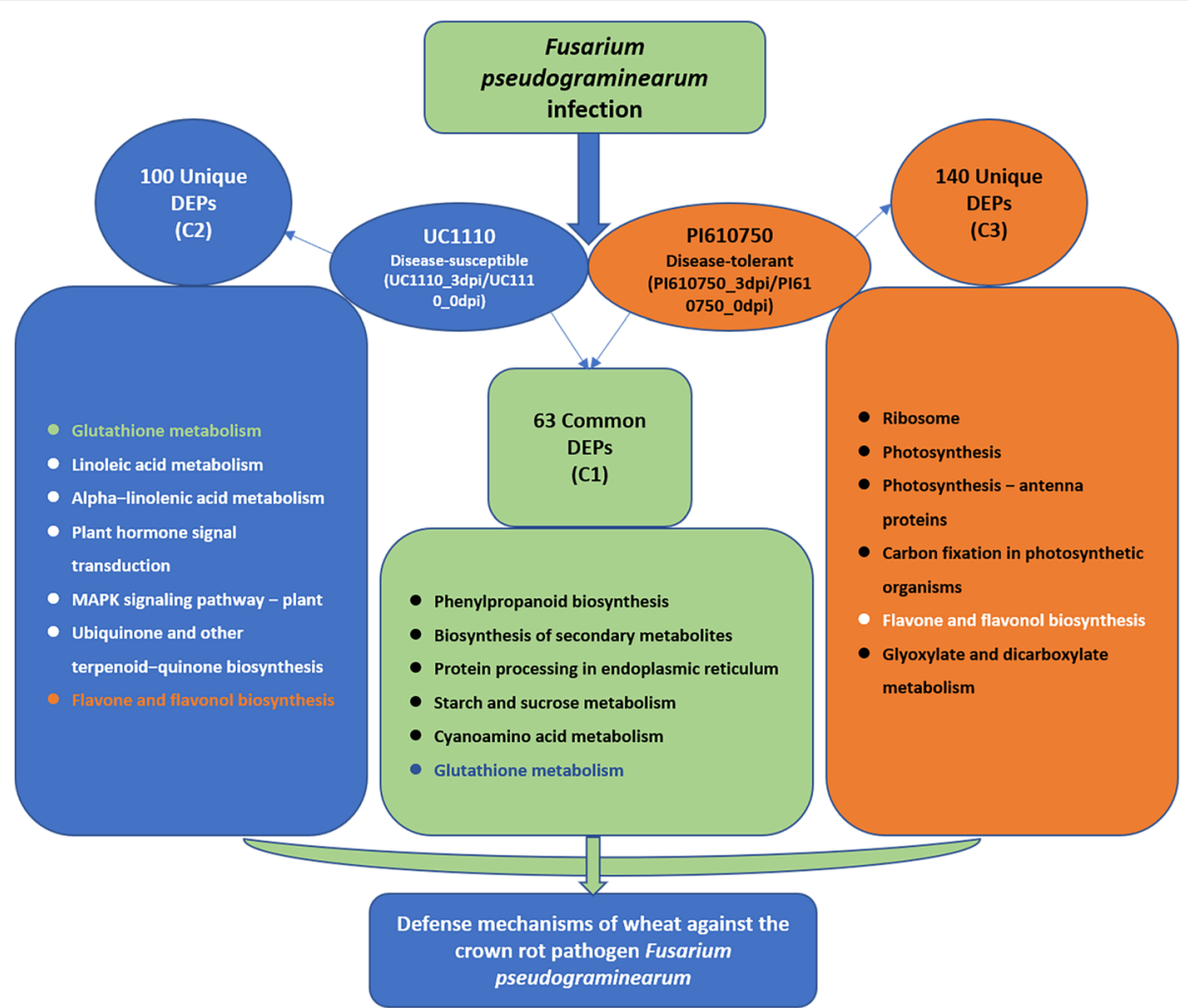

Fig. 8 Schematic model of the defense mechanisms of wheat against the crown rot pathogen F. pseudograminearum

photosynthesis is reduced after pathogen invasion, such as in barley infected with powdery mildew, potato infected with Phytophthora infestans, and soybean infected with Phytophthora sojae [50-52]. In this study, the specific DEPs in the comparison group of PI610750_3dpi/ PI610750_0dpi were significantly enriched in the three photosynthesis-related pathways of photosynthesis (osa00195), photosynthesis-antennary protein (osa00196), and carbon fixation (osa00710) (Fig. 6). The increase in the abundance of photosynthesis-related proteins may reflect the fact that photosynthesis provides a large amount of energy for plant defense. Thus, photosynthesis-related proteins in the disease-tolerant cultivar PI610750 play an important role in disease defense.

Previous studies have shown that sedoheptulose-1,7bisphosphatase, phosphoribulokinase, glyceraldehyde-3phosphate dehydrogenase, and ribulose 1,5-bisphosphate carboxylase/oxygenase (Rubisco) are involved in the Calvin cycle, and phosphoglycerate kinase participates in the glycolytic, gluconeogenic, and photosynthetic pathways $[53,54]$. It also has been reported that increasing the activity of sedoheptulose-1,7-bisphosphatase in transgenic tobacco plants can promote photosynthesis and growth from the early stages of development [55]. Rubisco is an enzyme complex in plants that is composed of eight large subunits and eight small subunits [56]. It has been reported that the abundance of the small and large subunits of Rubisco increased significantly in Zhongmu-1 $8 \mathrm{~h}$ after salt treatment [57]. An increase in the abundance of Rubisco large subunits and a decrease in small subunits have also been detected in nontransgenic wheat in response to drought $[58,59]$. It has been reported that Rubisco large subunits and ribose- 1 are down-regulated at 24 $\mathrm{h}$ post-inoculation and then up-regulated at 48 and $72 \mathrm{~h}$ post-inoculation [60].

In our study, we detected enriched sedoheptulose-1, 7-bisphosphatase, phosphoribulokinase, phosphoglycerate kinase, glyceraldehyde-3-phosphate dehydrogenase, Rubisco large subunits, and Rubisco small subunits in the carbon fixation pathway of photosynthetic organisms (osa00710) of PI610750. The increase in abundance of these DEPs indicated that photosynthesis plays a major role in the defense mechanisms of the disease-tolerant cultivar PI610750. However, these DEPs were not observed in the disease-susceptible cultivar UC1110. In summary, the disease-tolerant cultivar PI610750 may defend itself against disease by increasing its photosynthetic rate, thus providing energy for itself. 


\section{Conclusions}

Through TMT-based quantitative proteomic analysis, we confirmed that the physiological and biochemical responses of the wheat disease-tolerant cultivar PI610750 and disease-susceptible cultivar UC1110 were significantly different under $F$. pseudograminearum stress. Based on the cluster analysis results of the GO enrichment and KEGG pathway enrichment, the metabolic pathways of the wheat response to $F$. pseudograminearum stress may be complex. The disease-tolerant cultivar PI610750 and the susceptible cultivar UC1110 interacted with pathogens during the incubation period. Although these cultivars shared many of the same metabolic pathways, they also possessed unique pathways. The unique pathways in the susceptible cultivar UC1110 were mainly related to linoleic acid metabolism, plant hormone signal transduction, MAPK signaling pathway-plant, and ubiquinone biosynthesis, while the unique pathways in the disease-tolerant cultivar PI610750 were mainly related to photosynthesis, carbon fixation in photosynthetic organisms, flavone and flavonol biosynthesis, and glyoxylate and dicarboxylate metabolism. The DEPs in the seedling stem bases of the disease-susceptible cultivar UC1110 were mainly related to glutathione metabolism, nitrogen metabolism, and phenylpropane biosynthesis, whereas the DEPs in the seedling stem bases of the disease-tolerant cultivar PI610750 were mainly related to photosynthesis. This indicated that there are differences in the defense mechanism of the diseasetolerant wheat cultivar PI610750 and the diseasesusceptible cultivar UC1110 against $F$. pseudograminearum, which might provide a perspective for wheat genetic improvement and breeding.

\section{Methods \\ Experimental materials and inoculation}

The wheat cultivars UC1110 and PI610750, which were kindly provided by Prof. Jorge Dubcovsky from the University of California, Davis, were used in our experiments. A strong and aggressive F. pseudograminearum strain, WZ-8A (Accession: JN862232.1), which was kindly provided by Prof. Honglian Li from the College of Plant Protection of Henan Agricultural University, was used in this study. The cultivar UC1110 is susceptible to the predominant Chinese isolate WZ-8A of F. pseudograminearum, whereas the cultivar PI610750 is tolerant. UC1110 and PI610750 seeds were sterilized by immersion in $75 \%(\mathrm{w} / \mathrm{v})$ alcohol for $30 \mathrm{~s}$ and then thoroughly washed with distilled water. The sterilized seeds were cultivated in sterilized pots $(12 \mathrm{~cm} \times 17 \mathrm{~cm})$ with 2 $\mathrm{kg}$ sterilized soil (sand: soil $=2.5: 1$ ). The seedlings were maintained in a growth chamber at $25 / 20^{\circ} \mathrm{C}$ day/night temperatures under a $16 / 8 \mathrm{~h}$ light/dark photoperiod and $65 / 75 \%$ day/night relative humidity.

We infected one-week-old seedlings with $F$. pseudograminearum from approximately $20 \mathrm{~g}$ of millet matrix and used plants at $0 \mathrm{dpi}$ as the control, with untreated $3 \mathrm{dpi}$ seedlings used as the negative control. We collected the stem bases of the two wheat cultivars at $0,1,2$, and 3 dpi, until symptoms were visible, and then stored the samples at $-80^{\circ} \mathrm{C}$ until protein extraction. We performed three biological replicates per treatment (Fig. 9).

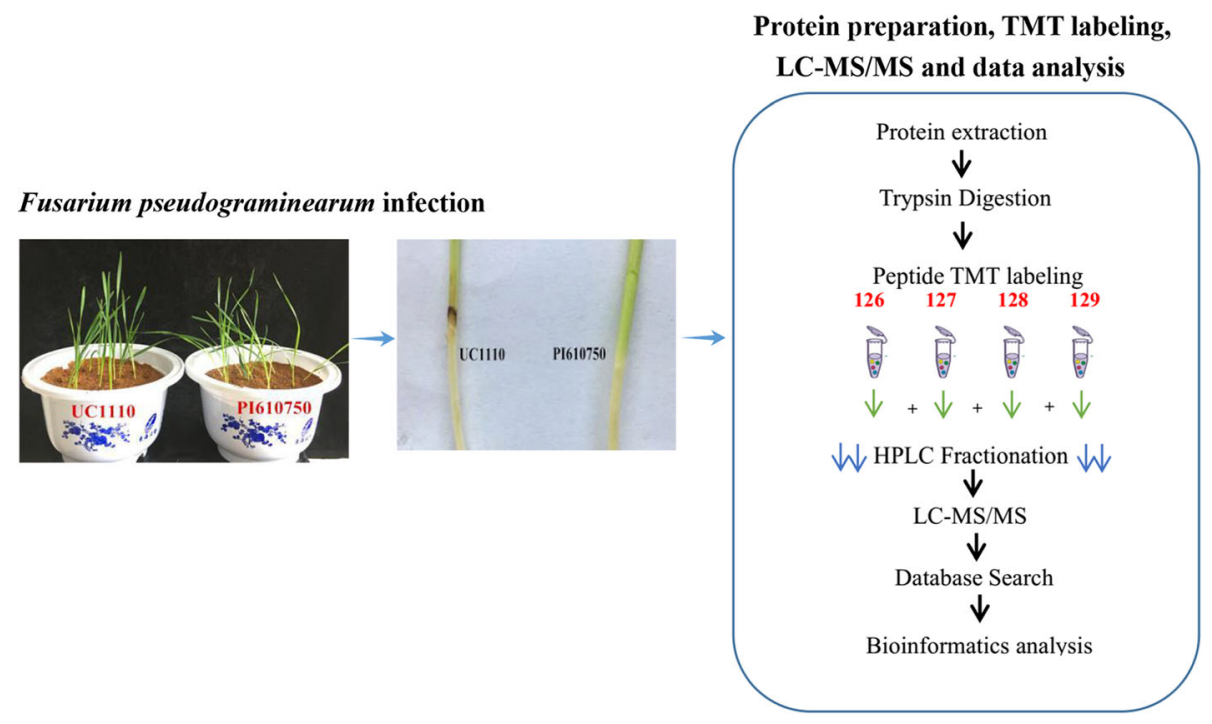

Fig. 9 Workflow for the characterization of defense mechanisms of wheat in the response to F. pseudograminearum infection using TMT-based quantitative proteomics technology. The stem bases of UC1110 and P1610750 seedlings were inoculated with the colonized grains 


\section{Measurements of plant morphological and physiological parameters}

We examined morphology using an Epson Expression 12000XL photo scanner (Seiko Epson Corporation, Suwa, Nagano, Japan) and analyzed morphological parameters using the Win-RHIZO (LA6400XL, Regent Instruments Inc., Quebec, Canada) system, including total root length, total root surface area, total root volume, average root diameter, number of root tips, and number of forks. We collected wheat roots and leaves at different times $(0,1,2$, and 3 dpi). Root activity was determined using the triphenyl tetrazolium chloride modified method, as described by Wang et al. and Cao et al. [61, 62]. We determined the content of total soluble sugar using a sulfuric acid-anthrone method [63, 64] and measured leaf chlorophyll content by spectrophotometry [65]. We measured the activity of enzymes related to stress, POD, SOD, and CAT according to previously described methods $[65,66]$ and measured the protein content using the Bradford method with bovine serum albumin (BSA) as the standard [67]. We determined MDA content according to previously described methods [65].

\section{Protein extraction}

Protein extraction, trypsin digestion, LC-MS (MS/MS) analysis, and the database search were performed with reference to previously reported methods [68-74], with some modifications. The sample was ground into a cell powder in liquid nitrogen. Then, the cell powder was moved to a $5-\mathrm{mL}$ centrifuge tube. During the next step, we added four volumes of lysis buffer (i.e., $8 \mathrm{M}$ urea, $1 \%$ Triton-100, $10 \mathrm{mM}$ dithiothreitol, and 1\% Protease Inhibitor Cocktail) to the cell powder. A high-intensity ultrasonic processor (Scientz, Zhejiang, China) was used to perform sonication three times on ice. We removed the remaining debris by centrifugation at $20,000 \mathrm{~g}$ at $4{ }^{\circ} \mathrm{C}$ for $10 \mathrm{~min}$. Finally, we precipitated the protein with cold $20 \%$ trichloroacetic acid for $2 \mathrm{~h}$ at $-20{ }^{\circ} \mathrm{C}$. The supernatant was discarded after centrifugation at 12,000 g at $4{ }^{\circ} \mathrm{C}$ for $10 \mathrm{~min}$. We washed the remaining precipitate with cold acetone three times. The protein was redissolved in $8 \mathrm{M}$ urea and a BCA kit was used to determine the protein concentration according to the manufacturer's instructions.

\section{Trypsin digestion}

For digestion, we used $5 \mathrm{mM}$ dithiothreitol for $30 \mathrm{~min}$ to reduce the protein solution at $56^{\circ} \mathrm{C}$. After that, at room temperature in darkness, the protein was alkylated with $11 \mathrm{mM}$ iodoacetamide for $15 \mathrm{~min}$. Then, we diluted the protein sample by adding $100 \mathrm{mM}$ TEAB until the urea concentration was less than $2 \mathrm{M}$. Finally, 1 : 50 and 1:100 trypsin-to-protein mass ratios were used for the first digestion overnight and a second $4 \mathrm{~h}$ digestion, respectively.

\section{LC-MS (MS/MS) analysis}

We dissolved the tryptic peptides in $0.1 \%$ formic acid (solvent A). Then, peptides were directly loaded onto a homemade reversed-phase analytical column $(15 \mathrm{~cm}$ length, $75 \mu \mathrm{m}$ i.d.). At a constant flow rate of $400 \mathrm{~nL} / \mathrm{min}$ on an EASY-nLC 1000 ultra-performance liquid chromatography (UPLC) system, the gradient of solvent B ( $0.1 \%$ formic acid in $98 \%$ acetonitrile) increased from 6 to $23 \%$ over $26 \mathrm{~min}$, increased from 23 to $35 \%$ in 8 min and climbed to $80 \%$ in $3 \mathrm{~min}$, and was then held at $80 \%$ for the last $3 \mathrm{~min}$. We subjected the peptides to an NSI source, then tandem mass spectrometry (MS/MS) in QExactiveTM Plus (ThermoFisher, Waltham, MA, USA) was coupled online to the UPLC. The $2.0 \mathrm{kV}$ electrospray voltage was applied. In the Orbitrap, the intact peptides were detected at a resolution of 70,000 , and the $\mathrm{m} / \mathrm{z}$ scan range was 350 to 1800 for a full scan. We then selected peptides for MS/MS using NCE set at 28. The fragments were then detected in the Orbitrap at a resolution of 17,500. We conducted a data-dependent procedure, which alternated between one MS scan followed by $20 \mathrm{MS} / \mathrm{MS}$ scans with a dynamic exclusion of $15.0 \mathrm{~s}$. We set at $5 E 4$ about the automatic gain control. The first mass was fixed at $100 \mathrm{~m} / \mathrm{z}$.

\section{Database search}

We used the Maxquant search engine (v.1.5.2.8) to process the resulting $\mathrm{MS} / \mathrm{MS}$ data. After that, we searched Tandem mass spectra against the UniProt Triticum aestivum database, which was concatenated with a reverse decoy database. As a cleavage enzyme, Trypsin/P was allowed up to two missing cleavages. For precursor ions in First search and in Main search, the mass tolerances were set at $20 \mathrm{ppm}$ and $5 \mathrm{ppm}$, respectively. We set the mass tolerance for fragment ions at $0.02 \mathrm{Da}$. Oxidation on Met and carbamidomethyl on Cys were specified as a variable modification and a fixed modification, respectively. We adjusted the false discovery rate (FDR) to $<1 \%$ and set the minimum score for peptides at $>40$. For the protein quantification, we selected TMT 6-plex method. The FDR was set at 0.01, and at least two peptides were required for protein groups quantification. With regard to the protein quantification, protein ratios were calculated through the median of only unique peptides of the protein. We normalized all peptide ratios by the median protein ratio. We used cutoff values of more than 1.50 -fold and less than 0.667 -fold to identify upregulated and down-regulated proteins using a $t$-test at $P<0.05$. 


\section{Bioinformatics analysis}

We derived the GO annotation proteome from the UniProt-GOA database (http://www.ebi.ac.uk/GOA/). We used the KEGG database to annotate protein pathways. We analyzed the protein-protein interactions for the identified proteins using the STRING v10.5 database (http://string-db.org) to determine their functions and pathways. We visualized the interaction network from STRING in Cytoscape (https://cytoscape.org/). We used a graph theoretical clustering algorithm [i.e., molecular complex detection (MCODE)] to analyze densely connected regions. MCODE is part of the plug-in toolkit of the network analysis and visualization software Cytoscape.

\section{Quantitative real-time reverse transcription-polymerase chain reaction}

The gene primers were designed by Online Primer 3.0 and are shown in Additional file 7. We extracted the total RNA from the wheat stem bases using the TaKaRa MiniBEST Plant RNA Extraction Kit (TaKaRa, Dalian, China). Reverse transcription of RNA was performed following the kit instructions (Promega Corp., Madison, WI, USA). The detailed method can be found in a previous publication [60]. The reproducibility of the results was guaranteed through three biological replicates. We performed the reactions in a CFX96 Real-Time PCR Detection System (Bio-Rad Laboratories, Inc., Hercules, CA, USA). We analyzed all data using CFX Manager Software (Bio-Rad Laboratories, Inc.). The relative expression levels were calculated using the $2^{-\Delta \Delta C T}$ method [75]. $\beta$-actin was used as an internal control gene.

\section{Statistical analyses}

We performed statistical analyses for morphological results across 10 biological replicates, for physiological and biochemical analyses across four biological replicates, and for proteomic analyses across three biological replicates. We performed analysis of variance using IBM SPSS Statistics 21.0 (IBM Corp., Armonk, NY, USA). Data are presented as means \pm standard deviation (SD) values. We determined the statistical significance using Student's $t$-tests at a $P<0.05$ threshold.

\section{Supplementary Information}

The online version contains supplementary material available at https://doi. org/10.1186/s12870-021-02853-6.

Additional file 1: Table S1. GO functional enrichment of 366 DEPS.

Additional file 2: Fig. S1. Distribution of all differentially expressed proteins in the KEGG pathway.

Additional file 3: Table S2. KEGG pathway enrichment of $C 1$.

Additional file 4: Table S3. KEGG pathway enrichment of $C 2$.

Additional file 5: Table S4. KEGG pathway enrichment of C3.
Additional file 6: Table S5. Protein-protein interaction network.

Additional file 7: Table S6. Primer sequence.

\section{Abbreviations}

TMT: Tandem mass tag; dpi: Days post inoculation; DEPs: Differentially expressed proteins; FCR: Fusarium crown rot; QTLs: Quantitative trait loci; CK: Untreated seedlings at 3 dpi; MDA: Malondialdehyde; SOD: Superoxide dismutase; POD: Peroxidase; CAT: Catalase; GO: Gene ontology; KEGG: Kyoto encyclopedia of genes and genomes; qRT-PCR: Quantitative real timepolymerase chain reaction; ProDH: Proline dehydrogenase; GSTs: Glutathione S-transferases; AOS: Allene oxide synthase; JA: Jasmonic acid; NADH-

GOGAT: NADH-dependent glutamate synthetase; PR proteins: Pathogenesisrelated proteins; SAR: System-acquired resistance; UPLC: Ultra-performance liquid chromatography; FDR: False discovery rate

\section{Acknowledgements}

We are grateful to Professor Honglian Li, Shengli Ding, and Feng Chen from Henan Agricultural University, and Professor Jorge Dubcovsky from the University of California, Davis for providing materials and guidance on the experiment. We also thank Dr. Xiaoyu Su, Haifeng Zhou, Moubiao Zhang, Jianzhao Duan and Yafan Zhao for writing assistance, and LetPub for the language editing of this manuscript.

\section{Authors' contributions}

QF, YXW, ZSM, and HDX designed the experiments. QF, XFD, HY, ZJM, and SM performed the experiments. QF and YXW analyzed the data. QF, YXW, $\mathrm{ZM}$, and HDX drafted and revised the manuscript. All authors read and approved the manuscript.

\section{Funding}

The work was supported by the National Key Research and Development Program "Science and Technology Innovation of High Grain Production Efficiency" of China (2018YFD0300701). The supporters played no role in the design of the study and collection, analysis, and interpretation of data and in writing the manuscript.

\section{Availability of data and materials}

All data analyzed in this study are included in this published article and its additional files. The mass spectrometry proteomics data have been deposited to the ProteomeXchange Consortium via the PRIDE partner repository (http://www.ebi.ac.uk/pride) with the dataset identifier PXD023314.

Ethics approval and consent to participate

Not applicable.

\section{Consent for publication}

Not applicable.

\section{Competing interests}

The authors declare that they have no competing interest.

Received: 3 October 2020 Accepted: 24 January 2021

Published online: 08 February 2021

\section{References}

1. Kazan K, Gardiner DM. Fusarium crown rot caused by Fusarium pseudograminearum in cereal crops: recent progress and future prospects. Mol Plant Pathol. 2018;19(7):1547-1562. doi.org/https://doi.org/10.1111/ mpp.12639

2. Knight NL, Sutherland MW. Histopathological assessment of Fusarium pseudograminearum colonization of cereal culms during crown rot infections. Plant Dis. 2016;100(2):252-259. doi.org/https://doi.org/10.1094/ PDIS-04-15-0476-RE

3. Liu CJ, Ogbonnaya FC. Resistance to Fusarium crown rot in wheat and barley: a review. Plant Breed. 2015;134(4):365-372. doi.org/https://doi.org/10. 1111/pbr.12274

4. Percy CD, Wildermuth GB, Sutherland MW. Symptom development proceeds at different rates in susceptible and partially resistant cereal seedlings infected with Fusarium pseudograminearum. Austral Plant Pathol. 2012;41(6):621-631. doi.org/https://doi.org/10.1007/s13313-012-0146-2 
5. Smiley RW, Gourlie JA, Easley SA, Patterson LM, Whittaker RG. Crop damage estimates for crown rot of wheat and barley in the Pacific Northwest. Plant Dis. 2005;89(6):595-604. doi.org/https://doi.org/10.1094/PD-89-0595

6. Murray GM, Brennan JP. Estimating disease losses to the Australian barley industry. Austral Plant Pathol. 2010;39:85-96. doi.org/https://doi.org/10.1071/ AP09064

7. Klein TA, Burgess LW, Ellison FW, Klein TA, Burgess LW, Ellison FW. The incidence and spatial patterns of wheat plants infected by Fusarium graminearum Group 1 and the effect of crown rot on yield. Aust J Agric Res. 1991;42:399-407. doi.org/https://doi.org/10.1071/AR9910399

8. Li HL, Yuan HX, Fu B, Xing XP, Tang WH: First report of Fusarium pseudograminearum causing crown rot of wheat in Henan, China. Plant Dis. 2012;96(7):1065. doi.org/https://doi.org/10.1094/PDIS-01-12-0007-PDN

9. Gardiner DM, Benfield AH, Stiller J, Stephen S, Aitken K, Liu C, et al. A High-resolution genetic map of the cereal crown rot pathogen Fusarium pseudograminearum provides a near-complete genome assembly. Mol Plant Pathol. 2018;19(1):217-226. doi.org/https://doi.org/ 10.1111/mpp.12519

10. Zhang XT, Gao F, Zhang F, Xie Y, Zhou L, Yuan HX, et al. The complete genomic sequence of a novel megabirnavirus from Fusarium pseudograminearum, the causal agent of wheat crown rot. Arch Virol. 2018; 163(11):3173-3175. doi.org/https://doi.org/10.1007/s00705-018-3970-z

11. Zhou HF, He XL, Wang S, Ma QZ, Sun B J, Ding SL, et al. Diversity of the Fusarium pathogens associated with crown rot in the Huanghuai wheatgrowing region of China. Environ Microbiol. 2019;21(8):2740-2754. doi.org/ https://doi.org/10.1111/1462-2920.14602

12. Chen LL, Geng XJ, Ma YM, Zhao JY, Chen WB, Xing XP, et al. The ER lumenal Hsp70 protein FpLhs1 is important for conidiation and plant infection in Fusarium pseudograminearum. Front Microbiol. 2019;10:1401. doi.org/https://doi.org/10.3389/fmicb.2019.01401

13. Chen LL, Ma YM, Zhao JY, Geng XJ, Chen WB, Ding SL, et al. The bZIP transcription factor FpAda1 is essential for fungal growth and conidiation in Fusarium pseudograminearum. Curr Genet. 2020;66(3):507-515. doi.org/ https://doi.org/10.1007/s00294-019-01042-1

14. Kang RJ, Li GN, Zhang MJ, Zhang PP, Wang LM, Zhang YS, et al. Expression of Fusarium pseudograminearum FpNPS9 in wheat plant and its function in pathogenicity. Curr Genet. 2020;66(1):229-243. doi.org/https://doi.org/10. 1007/s00294-019-01017-2

15. Pariyar SR, Erginbas-Orakci G, Dadshani S, Chijioke OB, Léon J, Dababat AA, et al. Dissecting the genetic complexity of Fusarium crown rot resistance in wheat. Sci Rep. 2020;10(1):3200. doi.org/https://doi.org/10.1038/s41598-02060190-4

16. Poole GJ, Smiley RW, Paulitz TC, Walker CA, Carter AH, See DR, Garland-Campbell K. Identification of quantitative trait loci (QTL) for resistance to Fusarium crown rot (Fusarium pseudograminearum) in multiple assay environments in the Pacific Northwestern US. Theor Appl Genet. 2012;125(1):91-107. doi.org/https://doi.org/10.1007/ s00122-012-1818-6

17. Ceoloni C, Forte P, Kuzmanović L, Tundo S, Moscetti I, De Vita P, et al. Cytogenetic mapping of a major locus for resistance to Fusarium head blight and crown rot of wheat on Thinopyrum elongatum 7EL and its pyramiding with valuable genes from a Th. ponticum homoeologous arm onto bread wheat 7DL. Theor Appl Genet. 2017;130(10):2005-2024. doi.org/ https://doi.org/10.1007/s00122-017-2939-8

18. Yang $X$, Pan $Y$, Singh $P K$, He $X$, Ren $Y$, Zhao L, et al. Investigation and genome-wide association study for Fusarium crown rot resistance in Chinese common wheat. BMC Plant Biol. 2019;19:153. doi.org/https://doi. org/10.1186/s12870-019-1758-2

19. Blum A, Benfield AH, Sørensen JL, Nielsen MR, Bachleitner S, Studt L, et al. Regulation of a novel Fusarium cytokinin in Fusarium pseudograminearum. Fungal Biol. 2019;123(3):255-266. doi.org/https://doi.org/10.1016/j.funbio. 2018.12 .009

20. Sørensen JL, Benfield AH, Wollenberg RD, Westphal K, Wimmer R, Nielsen $M R$, et al. The cereal pathogen Fusarium pseudograminearum produces a new class of active cytokinins during infection. Mol Plant Pathol. 2018;19(5): 1140-1154. doi.org/https://doi.org/10.1111/mpp.12593

21. Carlson R, Tugizimana F, Steenkamp PA, Dubery IA, Labuschagne N. Differential metabolic reprogramming in Paenibacillus Alvei-primed Sorghum bicolor seedlings in response to Fusarium pseudograminearum infection. Metabolites. 2019:9(7):150. doi.org/https://doi.org/10.3390/ metabo9070150
22. Desmond OJ, Edgar Cl, Manners JM, Maclean DJ, Schenk PM, Kazan K: Methyl jasmonate induced gene expression in wheat delays symptom development by the crown rot pathogen Fusarium pseudograminearum. Physiol Mol Plant Pathol. 2005;67(3-5):171-179. doi.org/https://doi.org/10. 1016/j.pmpp.2005.12.007

23. Desmond OJ, Manners JM, Schenk PM, Maclean DJ, Kazan K: Gene expression analysis of the wheat response to infection by Fusarium pseudograminearum. Physiol Mol Plant Pathol. 2008;73(1-3):40-47. doi.org/ https://doi.org/10.1016/j.pmpp.2008.12.001

24. Powell JJ, Carere J, Fitzgerald TL, Stiller J, Covarelli L, Xu Q, et al. The Fusarium crown rot pathogen Fusarium pseudograminearum triggers a suite of transcriptional and metabolic changes in bread wheat (Triticum aestivum L.). Ann Bot. 2017;119(5):853-867. doi.org/https://doi.org/10.1093/aob/ mcw207

25. Weltmeier F, Ehlert A, Mayer CS, Dietrich K, Wang X, Schutze K, et al. Combinatorial control of Arabidopsis proline dehydrogenase transcription by specific heterodimerisation of bZIP transcription factors. EMBO J. 2006;25(13):3133-3143. doi.org/https://doi.org/10.1038/sj.emboj. 7601206

26. Shen Y, Du J, Yue L, Zhan X: Proteomic analysis of plasma membrane proteins in wheat roots exposed to phenanthrene. Environ Sci Pollut Res. 2016;23(11):10863-10871. doi.org/https://doi.org/10.1007/s11356-016-6307-z

27. Wang $Y, X u$ L, Tang MJ, Jiang HY, Chen W, Zhang W, et al. Functional and integrative analysis of the proteomic profile of radish root under $\mathrm{Pb}$ exposure. Front Plant Sci. 2016;7:1871. doi.org/https://doi.org/10.3389/fpls. 2016.01871

28. Piero ARL, Mercurio V, Puglisi I, Petrone G: Gene isolation and expression analysis of two distinct sweet orange [Citrus sinensis L. (Osbeck)] tau-type glutathione transferases. Gene. 2009:443(1-2):143-150. doi.org/https://doi. org/10.1016/j.gene.2009.04.025

29. Yasir M, He SP, Sun GF, Geng XL, Pan Z, Gong WF, et al. A Genome-Wide association study revealed key SNPs/Genes associated with salinity stress tolerance in upland cotton. Genes. 2019;10(10):829. doi.org/https://doi.org/ 10.3390/genes10100829

30. Islam MDS, Choudhury M, Majlish AK, Islam T, Ghosh A. Comprehensive genome-wide analysis of Glutathione S-transferase gene family in potato (Solanum tuberosum L.) and their expression profiling in various anatomical tissues and perturbation conditions. Gene. 2018;639:149-162. doi.org/ https://doi.org/10.1016/j.gene.2017.10.007

31. Hause B, Maier W, Miersch $O$, Kramell R, Strack D. Induction of jasmonate biosynthesis in arbuscular mycorrhizal barley roots. Plant Physiol. 2002; 130(3):1213-1220. doi.org/https://doi.org/10.1104/pp.006007

32. Schaller F, Schaller A, Stintz A. Biosynthesis and metabolism of jasmonates. J Plant Growth Regul. 2005;23(3):179-199. doi.org/https://doi.org/10.1007/ s00344-004-0047-x

33. De Domenico S, Bonsegna S, Horres R, Pastor V, Taurino M, Poltronieri P, et al. Transcriptomic analysis of oxylipin biosynthesis genes and chemical profiling reveal an early induction of jasmonates in chickpea roots under drought stress. Plant Physiol Biochem. 2012;61:115-122. doi.org/https://doi. org/10.1016/j.plaphy.2012.09.009

34. Naqvi RZ, Zaidi SS, Mukhtar MS, Amin I, Mishra B, Strickler S, et al. Transcriptomic analysis of cultivated cotton Gossypium hirsutum provides insights into host responses upon whitefly-mediated transmission of cotton leaf curl disease. PLoS One. 2019;14(2):e0210011. doi.org/https://doi.org/10. 1371/journal.pone.0210011

35. Alazem M, Lin NS: Roles of plant hormones in the regulation of host-virus interactions. Plant Pathol. 2015;16(5):529-540. doi.org/https://doi.org/10. 1111/mpp.12204

36. Kojima S, Konishi N, Beier MP, Ishiyama K, Maru I, Hayakawa T, et al. NADHdependent glutamate synthase participated in ammonium assimilation in Arabidopsis root. Plant Signal Behav. 2014;9(8):e29402. doi.org/https://doi. org/10.4161/psb.29402

37. Mauriat M, Leplé JC, Claverol S, Bartholomé J, Negroni L, Richet N, et al. Quantitative proteomic and phosphoproteomic approaches for deciphering the signaling pathway for tension wood formation in poplar. J Proteome Res. 2015;14(8):3188-3203. doi.org/https://doi.org/10.1021/acs.jproteome. 5 b00140

38. Ma QP, Li H, Zou ZW, Arkorful E, Lv QR, Zhou QQ, et al. Transcriptomic analyses identify albino-associated genes of a novel albino tea germplasm 'Huabai 1'. Hortic Res. 2018;5:54. doi.org/https://doi.org/10. 1038/s41438-018-0053-y 
39. Reinprecht $Y$, Yadegari Z, Perry GE, Siddiqua M, Wright LC, McClean PE, et al. In silico comparison of genomic regions containing genes coding for enzymes and transcription factors for the phenylpropanoid pathway in Phaseolus vulgaris L. and Glycine max L. Merr. Front Plant Sci. 2013;4:317. doi. org/https://doi.org/10.3389/fpls.2013.00317

40. Morris JS, Caldo KMP, Liang S, Facchini PJ. PR10/Bet v1-like proteins as novel contributors to plant biochemical diversity. Chembiochem. 2020. doi.org/ https://doi.org/10.1002/cbic.202000354

41. Cao Y, Han Y, Meng D, Li D, Jin Q, Lin Y, et al. Structural, evolutionary, and functional analysis of the class iii peroxidase gene family in Chinese pear (Pyrus bretschneideri). Front Plant Sci. 2016;7:1874. doi.org/https://doi.org/10. 3389/fpls.2016.01874

42. Proietti S, Bertini L, Van der Ent S, Leon-Reyes A, Pieterse CM, Tucci M, et al. Cross activity of orthologous WRKY transcription factors in wheat and Arabidopsis. J Exp Bot. 2011;62(6):1975-1990. doi.org/https://doi.org/10. 1093/jxb/erq396

43. Hollbacher B, Schmitt AO, Hofer H, Ferreira F, Lackner P. Identification of proteases and protease inhibitors in allergenic and non-allergenic pollen. Int J Mol Sci. 2017;18(6):1199. doi.org/https://doi.org/10.3390/ ijms18061199

44. Breen S, Williams SJ, Outram M, Kobe B, Solomon PS. Emerging insights into the functions of pathogenesis-related protein 1. Trends Plant Sci. 2017;22(10):871-879. doi.org/https://doi.org/10.1016/j.tplants. 2017.06.013

45. Gamir J, Darwiche R, Van't Hof P, Choudhary V, Stumpe M, Schneiter R, et al. The sterol-binding activity of pathogenesis-related protein 1 reveals the mode of action of an antimicrobial protein. Plant J. 2017;89(3):502-509. doi. org/https://doi.org/10.1111/tpj.13398

46. Wu SW, Wang HW, Yang ZD, Kong LR. Expression comparisons of pathogenesis-related (PR) genes in wheat in response to infectioninfestation by Fusarium, Yellow dwarf virus (YDV) aphid-transmitted and Hessian Fly. J Integr Agric. 2014;13:926-936. doi.org/https://doi.org/10.1016/S20953119(13)60570-5.

47. Zhang JR, Wang F, Liang F, Zhang YJ, Ma LS, Wang HY, et al. Functional analysis of a pathogenesis-related thaumatin-like protein gene TaLr35PR5 from wheat induced by leaf rust fungus. BMC Plant Biol. 2018;18(1):76. doi. org/https://doi.org/10.1186/s12870-018-1297-2

48. Lu SW, Faris JD, Sherwood R, Friesen TL, Edwards MC. A dimeric PR-1-type pathogenesis-related protein interacts with ToxA and potentially mediates ToxA-induced necrosis in sensitive wheat. Mol Plant Pathol. 2014;15(7):650663. doi.org/https://doi.org/10.1111/mpp.12122

49. Wang XB, Chen XZ, Li JR, Zhou XX, Liu YT, Zhong LT, et al. Global analysis of lysine succinylation in patchouli plant leaves. Hortic Res. 2019;6:133. doi.org/ https://doi.org/10.1038/s41438-019-0216-5

50. Swarbrick PJ, Schulze-Lefert P, Scholes JD. Metabolic consequences of susceptibility and resistance (race-specific and broad-spectrum) in barley leaves challenged with powdery mildew. Plant Cell Environ. 2006;29(6): 1061-1076. doi.org/https://doi.org/10.1111/j.1365-3040.2005.01472.x

51. Restrepo S, Myers KL, Del PO, Martin GB, Hart AL, Buell CR, et al. Gene profiling of a compatible interaction between Phytophthora infestans and Solanum tuberosum suggests a role for carbonic anhydrase. Mol PlantMicrobe Interact. 2005;18:913-922. doi.org/https://doi.org/10.1094/MPMI-180913

52. Moy P, Qutob D, Chapman BP, Atkinson I, Gijzen M. Patterns of gene expression upon infection of soybean plants by Phytophthora sojae. Mol Plant-Microbe Interact. 2004;17:1051-1062. doi.org/https://doi.org/10.1094/ MPMI.2004.17.10.1051

53. Missihoun TD, Kotchoni SO, Bartels D. Aldehyde dehydrogenases function in the homeostasis of pyridine nucleotides in Arabidopsis thaliana. Sci Rep. 2018;8(1):2936. doi.org/https://doi.org/10.1038/s41598018-21202-6

54. Du DF, Gao X, Geng J, Li QY, Li L, Lv Q, et al. Identification of key proteins and networks related to grain development in wheat (Triticum aestivum L.) by comparative transcription and proteomic analysis of allelic variants in TaGW2-6A. Front Plant Sci. 2016;7:922. doi.org/https://doi.org/10.3389/fpls. 2016.00922

55. Lefebvre S, Lawson T, Zakhleniuk OV, Lloyd JC, Raines CA, Fryer M. Increased sedoheptulose-1,7-bisphosphatase activity in transgenic tobacco plants stimulates photosynthesis and growth from an early stage in development. Plant Physiol. 2005;138(1):451-460. doi.org/https://doi.org/10.1104/pp.104. 055046
56. Meng FJ, Luo QX, Wang QY, Zhang XL, Qi ZH, Xu FL, et al. Physiological and proteomic responses to salt stress in chloroplasts of diploid and tetraploid black locust (Robinia pseudoacacia L.). Sci Rep. 2016;6:23098. doi.org/https:// doi.org/10.1038/srep23098

57. Long RC, Li MN, Zhang TJ, Kang JM, Sun Y, Cong LL, et al. Comparative proteomic analysis reveals differential root proteins in Medicago sativa and Medicago truncatula in response to salt stress. Front Plant Sci. 2016;7:424. doi.org/https://doi.org/10.3389/fpls.2016.00424

58. Caruso G, Cavaliere C, Foglia P, Gubbiotti R, Samperi R, Lagana A. Analysis of drought responsive proteins in wheat (Triticum durum) by 2D-PAGE and MALDI-TOF mass spectrometry. Plant Sci. 2009;177(6):570-576. doi.org/ https://doi.org/10.1016/j.plantsci.2009.08.007

59. Merewitz EB, Gianfagna T, Huang B. Protein accumulation in leaves and roots associated with improved drought tolerance in creeping bentgrass expressing an ipt gene for cytokinin synthesis. J Exp Bot. 2011;62(15):53115333. doi.org/https://doi.org/10.1093/jxb/err166

60. Li J, Yang XW, Liu XH, Yu HB, Du CY, Li MD, et al. Proteomic analysis of the compatible interaction of wheat and powdery mildew (Blumeria graminis $f$. sp. tritici). Plant Physiol Biochem. 2017;111:234-243. doi.org/https://doi.org/ 10.1016/j.plaphy.2016.12.006

61. Wang $X$, Zhang W, Hao Z, Li X, Zhang Y, Wang S. Principles and techniques of plant physiological biochemical experiment. 3rd ed. Beijing: Higher Education Press; 2015.

62. Cao XC, Wu LL, Wu MY, Zhu CQ, Jin QY, Zhang JH. Abscisic acid mediated proline biosynthesis and antioxidant ability in roots of two different rice genotypes under hypoxic stress. BMC Plant Biol. 2020;20(1):198. doi.org/ https://doi.org/10.1186/s12870-020-02414-3

63. Li H, Sun Q, Zhao S, Zhang W. Principles and techniques of plant physiological biochemical experiment. 3rd ed. Beijing: Higher Education Press; 2000.

64. Yu JJ, Chen SX, Zhao Q, Wang T, Yang CP, Diaz C, et al. Physiological and proteomic analysis of salinity tolerance in Puccinellia tenuiflora. J Proteome Res. 2011;10(9):3852-3870. doi.org/https://doi.org/10.1021/pr101102p

65. Zhang LT, Xin ZY, Yu X, Ma C, Liang WW, Zhu MC, et al. Osmotic stress induced cell death in wheat is alleviated by tauroursodeoxycholic acid and involves endoplasmic reticulum stress-related gene expression. Front Plant Sci. 2017;8:667. doi.org/https://doi.org/10.3389/fpls.2017.00667

66. Su XY, Fan XC, Shao RX, Guo J, Wang YC, Yang JP, et al. Physiological and ITRAQ-based proteomic analyses reveal that melatonin alleviates oxidative damage in maize leaves exposed to drought stress. Plant Physiol Biochem. 2019;142:263-274. doi.org/https://doi.org/10.1016/j. plaphy.2019.07.012

67. Bradford MM. A rapid and sensitive method for the quantification of microgram quantities of protein utilizing the principles of protein dye binding. Anal Biochem. 1976;72(s1-2):248-254. doi.org/https://doi.org/10. 1016/0003-2697(76)90527-3.

68. Liang S, Xie M, Tang J, Wang M, Zhang D, Hou S. Proteomics reveals the effect of type I interferon on the pathogenicity of duck hepatitis A virus genotype 3 in Pekin ducks. Vet Microbiol. 2020;248:108813. doi.org/https:// doi.org/10.1016/j.vetmic.2020.108813

69. Zhang Z, Liu H, Bi X, Yu H, Xu Y, Chen Y, et al. Differential response of Phytophthora sojae zoospores to soybean seed exudates provides evidence of seed exudates participate in host resistance. Plant Soil. 2020;452(1-2): 601-614. doi.org/https://doi.org/10.1007/s11104-020-04607-z

70. Su H, Luo Y, Sun J, Liu X, Ling S, Xu B, et al. Transglutaminase 3 promotes skin inflammation in atopic dermatitis by activating monocyte-derived dendritic cells via DC-SIGN. J Invest Dermatol. 2020;140(2):370-9 e8. doi.org/ https://doi.org/10.1016/j.jid.2019.07.703

71. Zhang LT, Hu CZ, Zhang X, Qin Z, Yan ZG, Wei QQ, et al. Protein acetylation in mitochondria plays critical functions in the pathogenesis of fatty liver disease. BMC Genomics. 2020;21(1):435. doi.org/https://doi.org/10.1186/ s12864-020-06837-y

72. Yuan L, Wang J, Xie S, Zhao M, Nie L, Zheng Y, et al. Comparative proteomics indicates that redox homeostasis is involved in high- and lowtemperature stress tolerance in a novel Wucai (Brassica campestris L.) genotype. Int J Mol Sci. 2019;20(15). doi.org/https://doi.org/10.3390/ ijms20153760

73. Bai F, Tu T, Qin F, Ma Y, Liu N, Liu Y, et al. Quantitative proteomics of changes in succinylated proteins expression profiling in left appendages tissue from valvular heart disease patients with atrial fibrillation. Clin Chim Acta. 2019;495:345-354. doi.org/https://doi.org/10.1016/j.cca.2019.05.002 
74. Yuan JJ, Zhang Q, Gong CX, Wang FX, Huang JC, Yang GQ, et al. Young plasma ameliorates aging-related acute brain injury after intracerebral hemorrhage. Biosci Rep. 2019;39(5). doi.org/https://doi.org/10.1042/ bsr20190537

75. Livak KJ, Schmittgen TD. Analysis of relative gene expression data using real-time quantitative PCR and the $2^{-\Delta \Delta C T}$ Method. Methods. 2001;25(4): 402-408. doi.org/https://doi.org/10.1006/meth.2001.1262

\section{Publisher's Note}

Springer Nature remains neutral with regard to jurisdictional claims in published maps and institutional affiliations.

Ready to submit your research? Choose BMC and benefit from:

- fast, convenient online submission

- thorough peer review by experienced researchers in your field

- rapid publication on acceptance

- support for research data, including large and complex data types

- gold Open Access which fosters wider collaboration and increased citations

- maximum visibility for your research: over $100 \mathrm{M}$ website views per year

At $\mathrm{BMC}$, research is always in progress.

Learn more biomedcentral.com/submissions 